Asymmetric synthesis of D-fagomine and its diastereoisomers

Stephen G. Davies,* Ai M. Fletcher, Matthew S. Kennedy, Paul M. Roberts and James E. Thomson

Department of Chemistry, Chemistry Research Laboratory, University of Oxford, Mansfield Road, Oxford, OX1 3TA, U.K.

E-mail: steve.davies@chem.ox.ac.uk<smiles>OCC1NCCC(O)[C@@H]1O</smiles>

D-fagomine<smiles>OCC1NCCC(O)C1O</smiles>

D-4-epi-fagomine
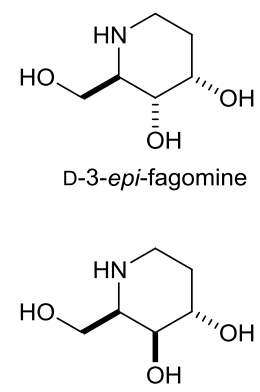


\title{
Asymmetric synthesis of D-fagomine and its diastereoisomers
}

Stephen G. Davies,* Ai M. Fletcher, Matthew S. Kennedy, Paul M. Roberts and James E. Thomson Department of Chemistry, Chemistry Research Laboratory, University of Oxford, Mansfield Road, Oxford, OX1 3TA, U.K.

E-mail: steve.davies@chem.ox.ac.uk

\begin{abstract}
A divergent strategy for the asymmetric syntheses of D-fagomine and three of its diastereoisomers has been developed. The diastereoselective conjugate addition of an enantiopure lithium amide to an $\alpha, \beta$-unsaturated ester was used as the key step to install the correct configuration required for the $\mathrm{C}(5)$-stereogenic centre within the targets. In situ enolate oxidation generated the corresponding anti- $\alpha$-hydroxy- $\beta$-amino ester, which possessed the correct configuration required for the $\mathrm{C}(4)$-stereogenic centre within both D-fagomine and D-3-epi-fagomine. Subsequent epimerisation of this key anti- $\alpha$-hydroxy- $\beta$-amino ester upon oxidation and diastereoselective reduction gave the corresponding syn- $\alpha$-hydroxy- $\beta$-amino ester, which possessed the correct configuration required for the $\mathrm{C}(4)$-stereogenic centre within both D-4-epi-fagomine and D-5-epifagomine. Elaboration of both $\alpha$-hydroxy- $\beta$-amino esters upon reduction to the corresponding aldehydes followed by aldol reaction generated the requisite $\mathrm{C}(3)$-stereogenic centres within the target compounds, then cyclisation and deprotection gave the enantiopure iminosugars in good overall yields, as single diastereoisomers (>99:1 dr).
\end{abstract}

Key words: D-fagomine, D-3-epi-fagomine, D-4-epi-fagomine, D-5-epi-fagomine, asymmetric synthesis, lithium amide

Dedicated to Professor Léon Ghosez in recognition of his major achievements in chemistry.

\section{Introduction}

D-Fagomine (1,2,5-trideoxy-1,5-imino-D-arabino-hexitol) $\mathbf{1}$ is a piperidine iminosugar which was first isolated from Japanese buckwheat, Fagopyrum esculentum, in 1974 by Koyama and Sagamura, ${ }^{1}$ and has more recently been isolated from a number of members of the Morus genus, ${ }^{2,3}$ Xanthocercis zambesiaca ${ }^{4}$ and the Moreton Bay chestnust Castanospermum australe. ${ }^{5}$ Its diastereoisomers, D-3-epi-fagomine (1,2,5trideoxy-1,5-imino-D-ribo-hexitol) 2 and L-5-epi-fagomine (1,2,5-trideoxy-1,5-imino-L-xylo-hexitol) ent-4 
have also been isolated from Nature, ${ }^{3,4,6,7}$ and this class of hydroxylated piperidines has been commonly targeted in total syntheses. ${ }^{3,8}$ D-Fagomine 1, along with other iminosugars including D-3-epi-fagomine 2 , have been reported to show antihyperglycemic effects in streptozotocin-induced diabetic mice. ${ }^{9}$ The inhibitory effects of D-fagomine 1 against $\alpha$-glucosidase and $\beta$-galactosidases have been reported, although D-3-epi-fagomine 2 was found to be more potent against these enzymes. Additionally, L-5-epi-fagomine ent-4 showed no significant inhibitory effects against any of the glycosidases used in the study. ${ }^{4}$ The synthetic analogue D-4-epi-fagomine $\mathbf{3}$ has been shown to inhibit some $\alpha$-galactosidases although its inhibitory potency was reduced over 6000-fold when compared to its 2-hydroxylated analogue, D-1deoxygalactonojirimycin. ${ }^{10}$ Given the important biological activity of these compounds, we sought to develop a common strategy for the asymmetric synthesis of all of the eight stereoisomers of fagomine (Fig. 1). It was envisaged that our diastereoselective aminohydroxylation methodology ${ }^{11}$ could be used to install the configuration required for the $\mathrm{C}(4)$ and $\mathrm{C}(5)$ stereogenic centres within these iminosugars, and that further elaboration of the resultant $\alpha$-hydroxy- $\beta$-amino esters via conversion to the corresponding aldehyde and ensuing aldol reaction would set the configuration required for the $\mathrm{C}(3)$ stereogenic centres within the target iminosugars, which could then be revealed upon cyclisation and deprotection. Our initial studies in this area, targeting all D-configured members of this family, are reported herein.

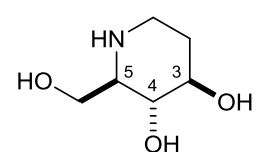

D-fagomine 1

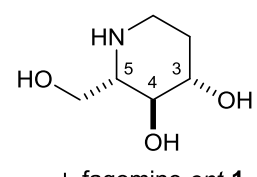

L-fagomine ent-1

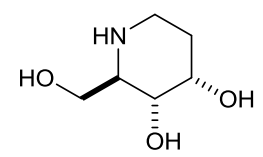

D-3-epi-fagomine 2

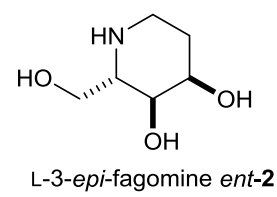

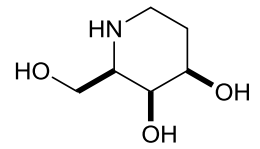

D-4-epi-fagomine 3

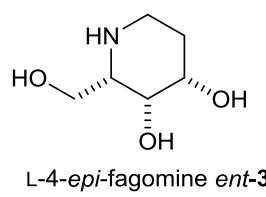

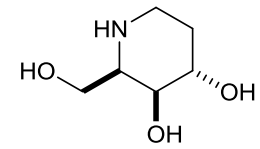

D-5-epi-fagomine 4

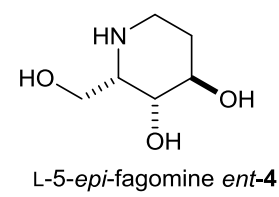

Fig. 1. The eight stereoisomers of fagomine.

\section{Results and Discussion}

Amino alcohol 9 was prepared according to our established procedure. ${ }^{12,13}$ Conjugate addition of enantiopure lithium amide ${ }^{11}(R)-6$ to $\alpha, \beta$-unsaturated ester 5 (which was prepared in $62 \%$ overall yield in 3 steps from cis-but-2-ene-1,4-diol) followed by oxidation of the intermediate lithium (Z)- $\beta$-amino enolate ${ }^{14}$ with (-)-camphorsulfonyloxaziridine [(-)-CSO] gave anti- $\alpha$-hydroxy- $\beta$-amino ester 7 in $77 \%$ yield and $>99: 1 \mathrm{dr}$. $O$-Benzylation of 7 was achieved in $92 \%$ yield and subsequent reduction of the ester moiety within 8 upon treatment with DIBAL-H gave the corresponding alcohol 9 in $68 \%$ yield as a single diastereoisomer (>99:1 dr). However, a superior overall yield was obtained upon sequential $O$-benzylation of 7 and 
immediate reduction of $\mathbf{8}$ (i.e., avoiding the purification of $\mathbf{8}$ ), which gave $\mathbf{9}$ in $87 \%$ yield (from 7) and >99:1 $\operatorname{dr}$ (Scheme 1).
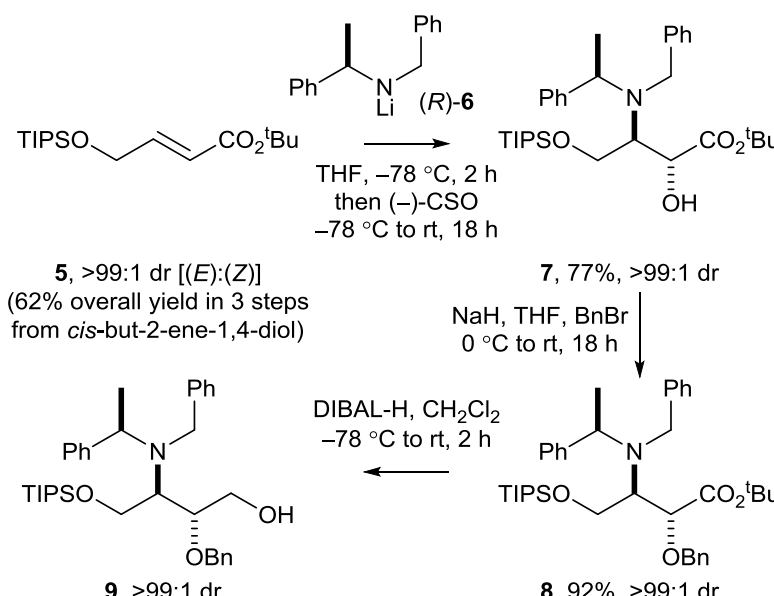

$68 \%$ (from 8 ), $87 \%$ (from 7 )

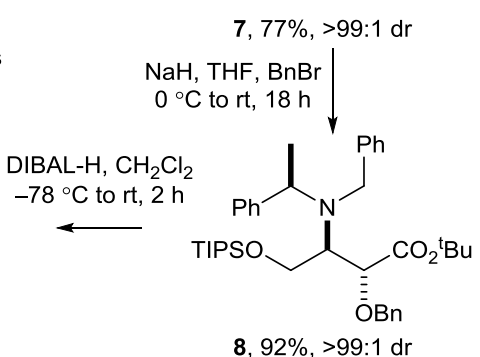

Scheme 1.

Oxidation of the primary hydroxyl functionality within $9,{ }^{15}$ followed by aldol reaction of the resultant aldehyde $\mathbf{1 0}$ with the lithium enolate derived from ethyl acetate, gave a 65:35 mixture of aldol products 11 and 12, respectively. Purification of the crude reaction mixture via flash column chromatography gave the major diastereoisomer 11 in 39\% yield and >99:1 dr; the minor diastereoisomer 12 was also isolated in 33\% yield and >99:1 dr, in addition to a mixed fraction comprising a 74:26 mixture of $\mathbf{1 1}$ and $\mathbf{1 2}$, respectively, in $12 \%$ combined yield. The configurations of $\mathbf{1 1}$ and $\mathbf{1 2}$ were established upon chemical correlation of the major diastereoisomer 11 to the known unsaturated amino alcohol $\mathbf{1 4},{ }^{12}$ which was synthesised in $51 \%$ yield and >99:1 dr upon addition of vinylmagnesium bromide to aldehyde 10. ${ }^{16}$ Hydroboration of the olefin within $\mathbf{1 4}$ gave diol 13 in 35\% yield (unoptimised) and >99:1 dr, and reduction of the ester moiety within 11 (>99:1 dr) upon treatment with $\mathrm{LiAlH}_{4}$ also gave $\mathbf{1 3}$ as a single diastereoisomer in $65 \%$ yield (Scheme 2). The spectroscopic data and specific rotations for these two samples of $\mathbf{1 3}$ were in complete accord, securing the configuration of $\mathbf{1 1}$. The configuration of its $\mathrm{C}(3)$-epimer 12 was confirmed upon chemical correlation to D-3-epi-fagomine 2 (vide infra). 


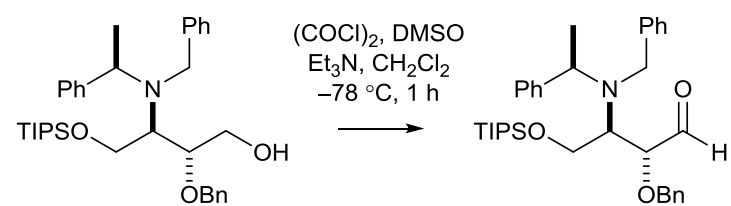

9, >99:1 dr

10 , not isolated

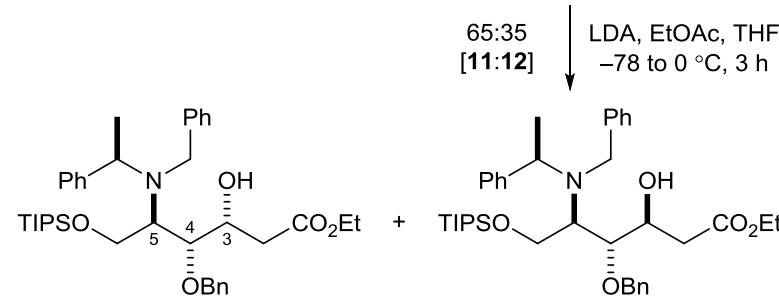

$11,39 \%,{ }^{a}>99: 1 \mathrm{dr}$

$12,33 \%,>99: 1 \mathrm{dr}$

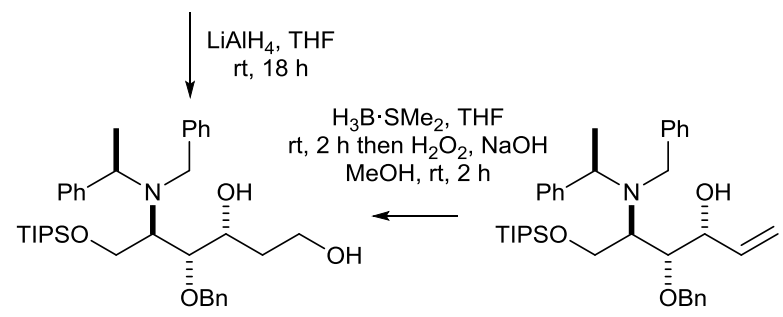

$13,>99: 1 \mathrm{dr}$

$65 \%$ (from 11), $35 \%$ (from 14 )

14, $>99: 1 d r$

Scheme 2. ${ }^{\text {a }} 74: 26$ mixture [11:12] was also isolated in $12 \%$ combined yield.

Elaboration of the major diastereoisomeric product $\mathbf{1 1}$ to D-fagomine $\mathbf{1}$ was achieved via hydrogenolysis in the presence of Pearlman's catalyst $\left[\mathrm{Pd}(\mathrm{OH})_{2} / \mathrm{C}\right]$, which effected complete $N$-debenzylation and cyclisation to the corresponding lactam $\mathbf{1 5},{ }^{17}$ although the $O$-benzyl protecting group was not removed under these conditions. Various attempts to remove this $O$-benzyl protecting group were evaluated with the most efficacious being reduction with sodium naphthalide, which gave 16 in 56\% isolated yield (from 11) as a single diastereoisomer (Scheme 3). The relative configuration of $\mathbf{1 6}$ was unambiguously confirmed via X-ray diffraction analysis (Fig. 2), ${ }^{18}$ and the assigned absolute $(R, R, R)$-configuration of 16 was confirmed upon refinement of a Flack $x$ parameter ${ }^{19}$ of $-0.02(6)$ for the structure $16,{ }^{20}$ which satisfies the criteria for a reliable assignment of absolute configuration of a material known to be enantiopure. Subsequent reduction of the amide functionality within 16 upon reduction with $\mathrm{BH}_{3}$ followed by $O$-desilylation under acidic conditions gave D-fagomine 1 in $65 \%$ yield and >99:1 dr after chromatographic purification (Scheme 3 ). The spectroscopic data, melting point and specific rotation of this sample of $\mathbf{1}$ \{mp $170-173{ }^{\circ} \mathrm{C} ;[\alpha]_{\mathrm{D}}^{25}+14.9(c$ 1.0 in $\left.\left.\mathrm{H}_{2} \mathrm{O}\right)\right\}$ were in good agreement with literature values $\left\{\right.$ lit. ${ }^{5} \mathrm{mp} 184-185{ }^{\circ} \mathrm{C}$; lit. ${ }^{1} \mathrm{mp} 186-188{ }^{\circ} \mathrm{C}$; lit. ${ }^{2,3}$

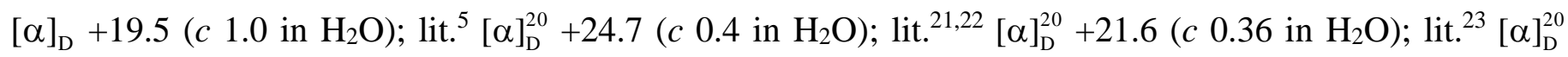
+17.9 ( $c 0.78$ in $\mathrm{H}_{2} \mathrm{O}$ ) ), which confirmed both the identity of this sample of $\mathbf{1}$ and also the assigned configurations of the synthetic precursors $\mathbf{1 1}, \mathbf{1 5}$ and $\mathbf{1 6 .}$ 


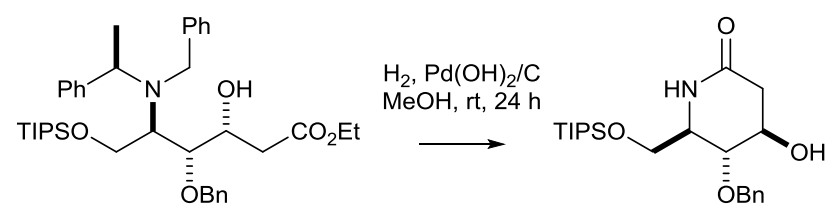

11, >99:1 dr

15, not isolated

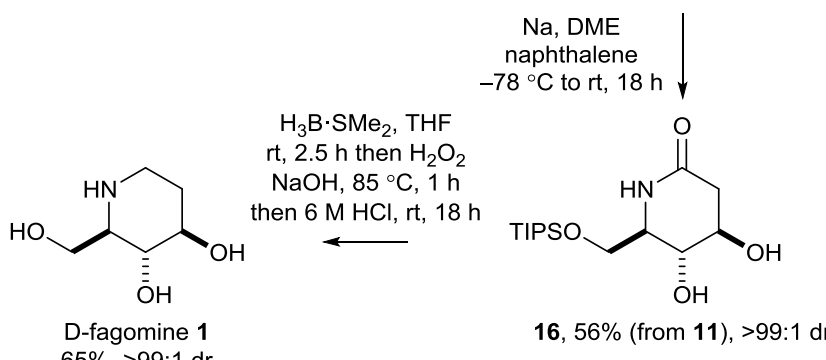

Scheme 3.

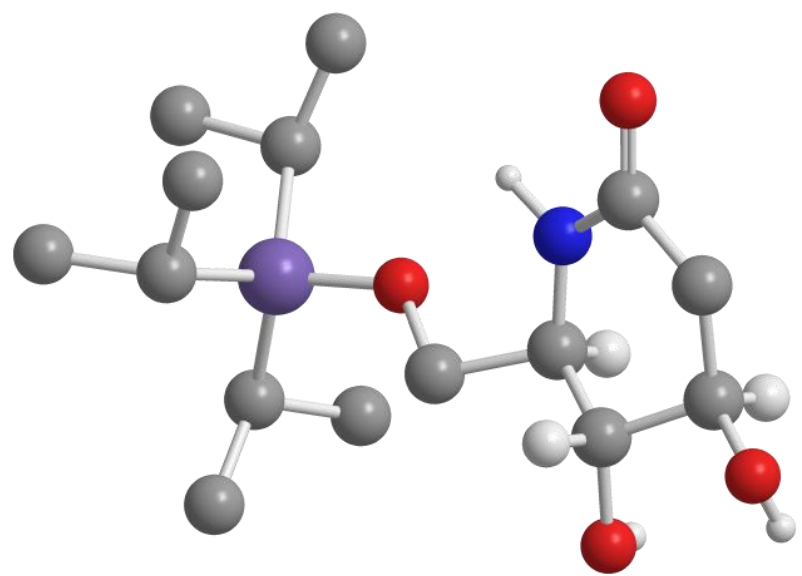

Fig. 2. X-ray crystal structure of $(R, R, R)-\mathbf{1 6}$ (selected $\mathrm{H}$ atoms have been omitted for clarity).

Similarly, D-3-epi-fagomine 2 was produced upon elaboration of the epimeric precursor 12. Hydrogenolysis of 12 effected $N$-debenzylation and cyclisation to give lactam 17, and treatment of 17 with sodium naphthalide gave 18 in 76\% yield (from 12) and >99:1 dr. Reduction of $\mathbf{1 8}$ with $\mathrm{BH}_{3}$ and $O$-desilylation then gave D-3-epi-fagomine 2 in 52\% yield as a single diastereoisomer (Scheme 4). The spectroscopic data, melting point and specific rotation of this sample of $2\left\{\mathrm{mp} 188-191{ }^{\circ} \mathrm{C} ;[\alpha]_{\mathrm{D}}^{25}+62.8\left(c 1.0\right.\right.$ in $\left.\left.\mathrm{H}_{2} \mathrm{O}\right)\right\}$ were in good agreement with literature values $\left\{\right.$ lit. $^{8 \mathrm{~d}} \mathrm{mp} 220-222{ }^{\circ} \mathrm{C}$; lit. ${ }^{3,4,6}[\alpha]_{\mathrm{D}}+69.0\left(c 0.5\right.$ in $\left.\mathrm{H}_{2} \mathrm{O}\right)$; lit. ${ }^{8 \mathrm{~d}}[\alpha]_{\mathrm{D}}^{26}$ $+74.4\left(c 0.95\right.$ in $\left.\mathrm{H}_{2} \mathrm{O}\right)$ ) , which confirmed both the identity of this sample of 2 and also the assigned configurations of the synthetic precursors 12,17 and 18 . 


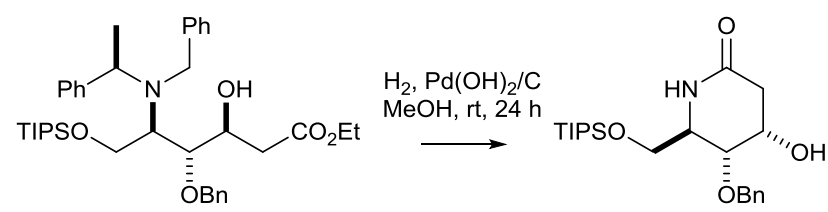

12, $>99: 1 \mathrm{dr}$

17, not isolated

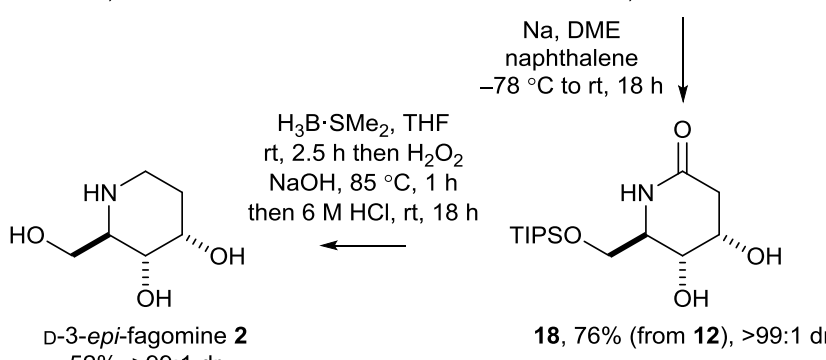

Scheme 4.

The two remaining D-configured diastereoisomers, D-4-epi-fagomine $\mathbf{3}$ and D-5-epi-fagomine $\mathbf{4}$, were targeted next via preparation of the epimeric syn- $\alpha$-hydroxy- $\beta$-amino ester $\mathbf{2 0}$ following an oxidation/diastereoselective reduction protocol ${ }^{24}$ and elaboration via an analogous strategy. Swern oxidation of 7 gave the intermediate ketone 19, and reduction of $\mathbf{1 9}$ with $\mathrm{NaBH}_{4}$ in $\mathrm{MeOH}$ at $-20{ }^{\circ} \mathrm{C}$ gave a 93:7 mixture of syn-20 and anti-7, respectively, from which $\mathbf{2 0}$ was isolated in 55\% yield and >99:1 dr. $O$-Benzylation of $\mathbf{2 0}$ and subsequent reduction of the ester moiety upon treatment with DIBAL-H gave $\mathbf{2 1}$ in 95\% yield as a single diastereoisomer. However, a superior overall yield was obtained upon sequential oxidation of alcohol 7, diastereoselective reduction of ketone 19, $O$-benzylation of $\mathbf{2 0}$, and subsequent ester reduction (i.e., avoiding the purification of intermediate $\mathbf{2 0}$ ), which gave $\mathbf{2 1}$ as a single diastereoisomer (>99:1 dr) in $85 \%$ overall yield (from 7). Oxidation of the primary hydroxyl functionality within 21, followed by aldol reaction of the resultant aldehyde with the lithium enolate derived from ethyl acetate, gave a 46:54 mixture of aldol products $\mathbf{2 2}$ and $\mathbf{2 3}$, respectively. Purification of the crude reaction mixture via flash column chromatography gave an inseparable 46:54 mixture of diastereoisomers $\mathbf{2 2}$ and $\mathbf{2 3}$, respectively, in $72 \%$ combined yield (Scheme 5). 

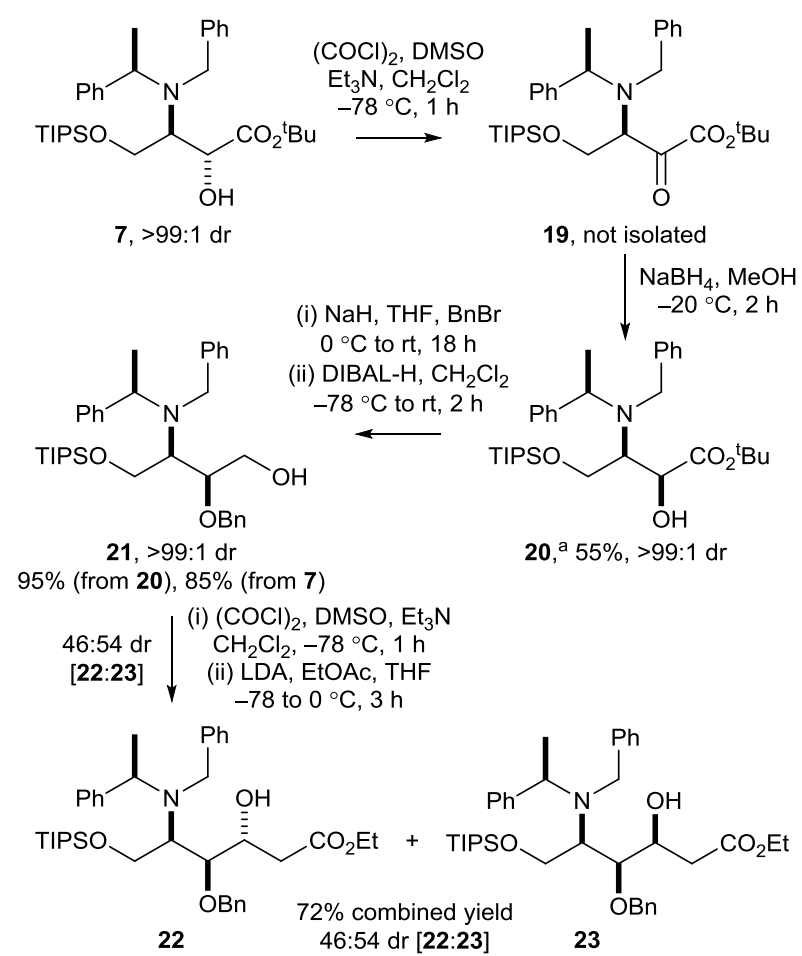

Scheme 5. ${ }^{\text {a }} 88: 12$ mixture [20:7] was also isolated in 32\% combined yield.

Treatment of the 46:54 mixture of $\mathbf{2 2}$ and $\mathbf{2 3}$, respectively, under hydrogenolytic conditions promoted $\mathrm{N}$-debenzylation and cyclisation to an $\sim 40: 60$ mixture of the epimeric lactams $\mathbf{2 4}$ and $\mathbf{2 5} .{ }^{25}$ Purification of this mixture via flash column chromatography gave $\mathbf{2 4}$ in $31 \%$ yield and $>99: 1 \mathrm{dr}$, and 25 in $35 \%$ yield and >99:1 dr (Scheme 6). The relative configuration of 25 was unambiguously established via X-ray diffraction analysis (Fig. 3), ${ }^{18,20}$ and the assigned absolute (4S,5S,6R)-configuration of $\mathbf{2 5}$ was confirmed upon refinement of a Flack $x$ parameter ${ }^{19}$ of $+0.10(8)$; this analysis therefore also secured the configuration of the epimer 24, and the synthetic precursors 20, 21 and 23. In both cases, reduction of the amide functionalities within 24 and 25 was followed by $O$-desilylation under acidic conditions to give D-4-epi-fagomine $3\left\{[\alpha]_{\mathrm{D}}^{25}\right.$ +13.9 (c 1.0 in $\left.\left.\mathrm{CHCl}_{3}\right)\right\}$ and D-5-epi-fagomine $4\left\{[\alpha]_{\mathrm{D}}^{25}+8.0\left(c 1.0\right.\right.$ in $\left.\left.\mathrm{H}_{2} \mathrm{O}\right)\right\}$, respectively, as single diastereoisomers (>99:1 dr) in 46 and $43 \%$ yield (Scheme 6). The spectroscopic data and specific rotations of these samples of $\mathbf{3}$ and $\mathbf{4}$ were in good agreement with literature values ${ }^{26}$ \{for D-4-epi-fagomine $\mathbf{3}$ : lit. ${ }^{8 b}$ $[\alpha]_{\mathrm{D}}^{25}+11.7\left(c 1.8\right.$ in $\left.\mathrm{H}_{2} \mathrm{O}\right)$; lit. $^{8 \mathrm{~g}}[\alpha]_{\mathrm{D}}^{25}+10.4\left(c 1.4\right.$ in $\left.\mathrm{H}_{2} \mathrm{O}\right)$; lit. $^{8 \mathrm{~d}}[\alpha]_{\mathrm{D}}^{22}+10.2\left(c 1.42\right.$ in $\left.\mathrm{H}_{2} \mathrm{O}\right)$; lit. ${ }^{3}[\alpha]_{\mathrm{D}}$

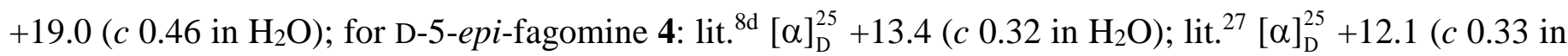
$\left.\mathrm{H}_{2} \mathrm{O}\right)$ \}, which confirmed both the identities of these samples of $\mathbf{3}$ and $\mathbf{4}$ and also the assigned configurations of the synthetic precursors $\mathbf{2 0 - 2 5}$. 

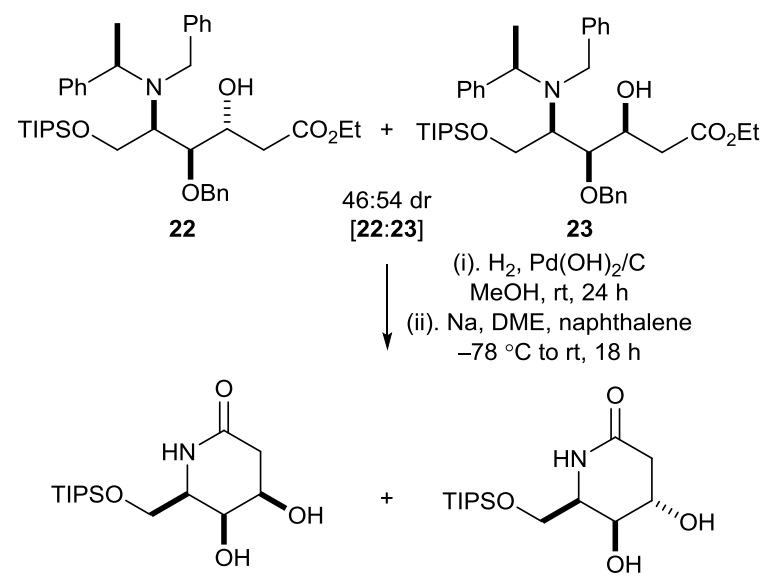

24, 31\%, >99:1 dr

$\mathrm{H}_{3} \mathrm{~B} \cdot \mathrm{SMe}_{2}$, THF rt, $2.5 \mathrm{~h}$ then $\mathrm{H}_{2} \mathrm{O}_{2}$ $\mathrm{NaOH}, 85^{\circ} \mathrm{C}, 1 \mathrm{~h}$

$\checkmark$ then $6 \mathrm{M} \mathrm{HCl}, \mathrm{rt}, 18 \mathrm{~h}$<smiles>OC[C@H]1NCCC(O)[C@@H]1O</smiles>

D-4-epi-fagomine 3 $46 \%,>99: 1 \mathrm{dr}$
25, 35\%, >99:1 dr

$\mathrm{H}_{3} \mathrm{~B} \cdot \mathrm{SMe}_{2}$, THF rt, $2.5 \mathrm{~h}$ then $\mathrm{H}_{2} \mathrm{O}_{2}$ $\mathrm{NaOH}, 85^{\circ} \mathrm{C}, 1 \mathrm{~h}$

then $6 \mathrm{M} \mathrm{HCl}, \mathrm{rt}, 18 \mathrm{~h}$

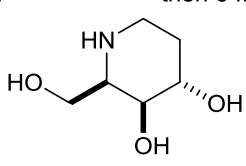

D-5-epi-fagomine 4 $43 \%,>99: 1 \mathrm{dr}$

Scheme 6.

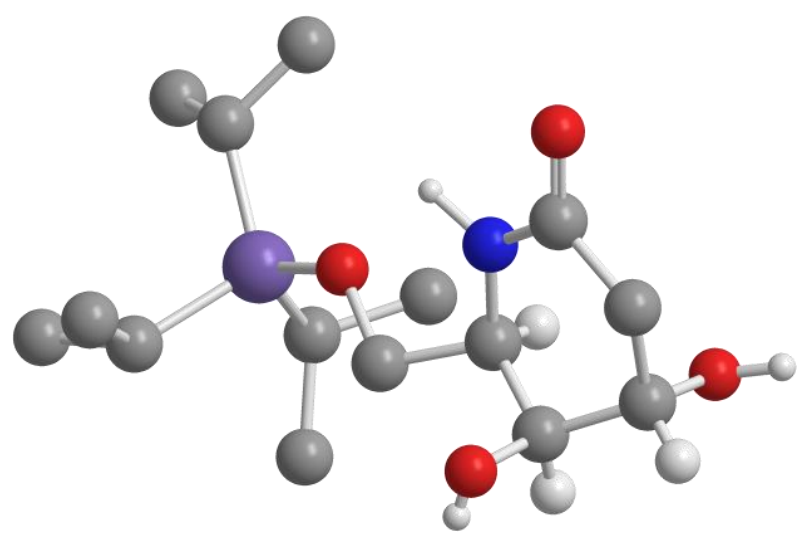

Fig. 3. X-ray crystal structure of $(4 S, 5 S, 6 R)-25$ (selected $\mathrm{H}$ atoms have been omitted for clarity).

\section{Conclusion}

In conclusion, a divergent strategy for the asymmetric syntheses of D-fagomine, D-3-epi-fagomine, D-4-epifagomine and D-5-epi-fagomine has been developed. The diastereoselective aminohydroxylation of an $\alpha, \beta$-unsaturated ester upon conjugate addition of lithium $(R)-N$-benzyl- $N$-( $\alpha$-methylbenzyl)amide followed by in situ enolate oxidation with (-)-camphorsulfonyloxaziridine gave an enantiopure anti- $\alpha$-hydroxy- $\beta$ amino ester [with the correct configuration required for the $\mathrm{C}(4)$ and $\mathrm{C}(5)$ stereogenic centres within D-fagomine and D-3-epi-fagomine], and epimerisation of this substrate gave the corresponding syn- $\alpha$ hydroxy- $\beta$-amino ester [with the correct configuration required for the $C(4)$ and $C(5)$ stereogenic centres within D-4-epi-fagomine and D-5-epi-fagomine]. Elaboration of both of these $\alpha$-hydroxy- $\beta$-amino esters upon reduction to the corresponding aldehydes followed by aldol reaction with the lithium enolate derived 
from ethyl acetate generated the C(3) stereogenic centres within the target iminosugars. Finally, cyclisation and deprotection gave the enantiopure iminosugars in good overall yields, as single diastereoisomers in each case. This strategy is also applicable to the asymmetric syntheses of the L-configured antipodes, by employing lithium $(S)$ - $N$-benzyl- $N$-( $\alpha$-methylbenzyl)amide and (+)-camphorsulfonyloxaziridine in the initial aminohydroxylation step. The syntheses of L-fagomine, L-3-epi-fagomine, L-4-epi-fagomine and L-5-epifagomine are ongoing within our laboratory, and the complete set of eight stereoisomers will consequently be subjected to biological evaluation.

\section{Experimental Section}

\subsection{General experimental details}

All reactions involving organometallic or other moisture-sensitive reagents were carried out under a nitrogen atmosphere using standard vacuum line techniques and glassware that was flame dried and cooled under nitrogen before use. Solvents were dried according to the procedure outlined by Grubbs and co-workers. ${ }^{28}$ BuLi was purchased as a solution in hexanes and titrated against diphenylacetic acid before use. All other reagents were used as supplied without prior purification. Organic layers were dried over $\mathrm{Na}_{2} \mathrm{SO}_{4}$. Thin layer chromatography was performed on aluminium plates coated with $60 \mathrm{~F}_{254}$ silica. Plates were visualised using UV light $(254 \mathrm{~nm}), 1 \%$ aq $\mathrm{KMnO}_{4}$ or Dragendorff's reagent. Flash column chromatography was performed on Kieselgel 60 silica. Melting points are uncorrected. Specific rotations are reported in $10^{-1} \mathrm{deg} \mathrm{cm}^{2} \mathrm{~g}^{-1}$ and concentrations in g/100 mL. IR spectra were recorded using an ATR module. Selected characteristic peaks are reported in $\mathrm{cm}^{-1}$. NMR spectra were recorded in the deuterated solvent stated. Spectra were recorded at $\mathrm{rt}$ unless otherwise stated. The field was locked by external referencing to the relevant deuteron resonance. ${ }^{1} \mathrm{H}-{ }^{1} \mathrm{H}$ COSY, ${ }^{1} \mathrm{H}^{-13} \mathrm{C} \mathrm{HMQC}$, and ${ }^{1} \mathrm{H}^{-13} \mathrm{C} \mathrm{HMBC}$ analyses were used to establish atom connectivity. Accurate mass measurements were run on a TOF spectrometer internally calibrated with polyalanine.

\section{2. tert-Butyl (E)-4-(triisopropylsilyloxy)but-2-enoate 5}

Step 1: TIPSCl (61.2 mL, $286 \mathrm{mmol})$ was added to a stirred solution of cis-2-butene-1,4-diol (12.0 g, 136 mmol), imidazole (27.8 g, $409 \mathrm{mmol})$ and DMAP (50 mg, cat.) in $\mathrm{CH}_{2} \mathrm{Cl}_{2}(300 \mathrm{~mL})$ at $\mathrm{rt}$, and the resultant mixture was stirred at $\mathrm{rt}$ for $18 \mathrm{~h}$ then concentrated in vacuo. The residue was dissolved in $\mathrm{Et}_{2} \mathrm{O}(400 \mathrm{~mL})$ and the resultant solution was washed with $1.0 \mathrm{M} \mathrm{aq} \mathrm{HCl}(200 \mathrm{~mL})$, then dried and concentrated in vacuo. Step 2: $\mathrm{O}_{3}$ was bubbled through a stirred, degassed solution of the residue from the previous step in $\mathrm{CH}_{2} \mathrm{Cl}_{2}$ $\left(500 \mathrm{~mL}\right.$ ) at $-78{ }^{\circ} \mathrm{C}$ until a blue colouration was apparent, then the reaction mixture was purged with $\mathrm{O}_{2}$ until 
it became colourless. $\mathrm{Me}_{2} \mathrm{~S}(10 \mathrm{~mL})$ was added dropwise and the resultant mixture was allowed to warm to $\mathrm{rt}$ over $18 \mathrm{~h}$. The reaction mixture was concentrated in vacuo, the residue was dissolved in $\mathrm{Et}_{2} \mathrm{O}(300 \mathrm{~mL})$ and the resultant solution was washed with $\mathrm{H}_{2} \mathrm{O}(200 \mathrm{~mL})$, then dried and concentrated in vacuo.

Step 3: $\mathrm{LiCl}(69.2 \mathrm{~g}, 1.63 \mathrm{~mol})$, tert-butyl diethylphosphonoacetate $(82.2 \mathrm{~g}, 326 \mathrm{mmol})$ and ${ }^{\mathrm{i}} \operatorname{Pr}_{2} \mathrm{NEt}(52.1$ $\mathrm{mL}, 299 \mathrm{mmol}$ ) were added to a stirred solution of the residue from the previous step in $\mathrm{MeCN}(500 \mathrm{~mL})$ and the resultant mixture was stirred at $\mathrm{rt}$ for $72 \mathrm{~h}$, then $\mathrm{H}_{2} \mathrm{O}(300 \mathrm{~mL})$ was added. The aqueous layer was extracted with EtOAc $(3 \times 300 \mathrm{~mL})$ and the combined organic extracts were dried and concentrated in vacuo. Purification via flash column chromatography (eluent $30-40{ }^{\circ} \mathrm{C}$ petroleum ether/Et $2 \mathrm{O}, 50: 1$ ) gave 5 as colourless oil $(52.7 \mathrm{~g}, 62 \%,>95: 5 \mathrm{dr}){ }^{29} \delta_{\mathrm{H}}\left(400 \mathrm{MHz}, \mathrm{CDCl}_{3}\right) 1.04-1.10\left(21 \mathrm{H}, \mathrm{m}, \mathrm{Si}\left(\mathrm{CHMe}_{2}\right)_{3}\right), 1.50$ (9H, s, CMe $), 4.41\left(2 \mathrm{H}, \mathrm{dd}, J 3.4,2.3, \mathrm{C}(4) H_{2}\right), 6.06(1 \mathrm{H}, \mathrm{dt}, J 15.4,2.3, \mathrm{C}(2) H), 6.90(1 \mathrm{H}, \mathrm{dt}, J 15.4,3.4$, $\mathrm{C}(3) H)$.

\section{3. tert-Butyl $(R, R, R)-2$-hydroxy-3-[ $N$-benzyl- $N$-( $\alpha$-methylbenzyl)amino]-4-}

\section{(triisopropylsilyloxy)butanoate 7}

BuLi (2.2 M in hexanes, $46.1 \mathrm{~mL}, 101 \mathrm{mmol})$ was added dropwise to a stirred solution of $(R)$ - $N$-benzyl- $N$ ( $\alpha$-methylbenzyl)amine $(22.1 \mathrm{~g}, 105 \mathrm{mmol},>99: 1 \mathrm{er})$ in THF $(170 \mathrm{~mL})$ at $-78^{\circ} \mathrm{C}$ and the resultant mixture was stirred at $-78{ }^{\circ} \mathrm{C}$ for $30 \mathrm{~min}$. A solution of 5 (20.6 g, $\left.65.4 \mathrm{mmol},>95: 5 \mathrm{dr}\right)$ in THF (170 mL) was then added and the resultant mixture was stirred at $-78^{\circ} \mathrm{C}$ for $2 \mathrm{~h}$. (-)-CSO $(30.0 \mathrm{~g}, 131 \mathrm{mmol})$ was added then the resultant mixture was allowed to warm to $\mathrm{rt}$ over $18 \mathrm{~h}$. Satd aq $\mathrm{NH}_{4} \mathrm{Cl}(30 \mathrm{~mL})$ was added and the reaction mixture was stirred at $\mathrm{rt}$ for $5 \mathrm{~min}$, then concentrated in vacuo. The residue was partitioned between $\mathrm{CH}_{2} \mathrm{Cl}_{2}(400 \mathrm{~mL})$ and $10 \%$ aq citric acid $(400 \mathrm{~mL})$, and the aqueous layer was extracted with $\mathrm{CH}_{2} \mathrm{Cl}_{2}$ $(3 \times 200 \mathrm{~mL})$. The combined organic layers were washed sequentially with satd aq $\mathrm{NaHCO}_{3}(300 \mathrm{~mL})$ and brine $(300 \mathrm{~mL})$, then dried and concentrated in vacuo. The residue was dissolved in $\mathrm{Et}_{2} \mathrm{O}(200 \mathrm{~mL})$, filtered and the filtrate was concentrated in vacuo to give a complex mixture. Purification via flash column chromatography (eluent $30-40{ }^{\circ} \mathrm{C}$ petroleum ether/Et $\left.2 \mathrm{O}, 30: 1\right)$ gave 7 as a colourless viscous oil (27.9 $\mathrm{g}$,

$77 \%,>95: 5 \mathrm{dr}) ;{ }^{13}[\alpha]_{\mathrm{D}}^{25}-35.7\left(c 1.0\right.$ in $\left.\mathrm{CHCl}_{3}\right)$; $\left\{\right.$ lit. ${ }^{13}$ for ent-7: $[\alpha]_{\mathrm{D}}^{20}+37.4\left(c 1.1\right.$ in $\left.\left.\mathrm{CHCl}_{3}\right)\right\} ; \delta_{\mathrm{H}}(400 \mathrm{MHz}$, $\left.\mathrm{CDCl}_{3}\right)$ 1.02-1.10 (21H, m, Si(CHMe $\left.)_{3}\right), 1.35(1 \mathrm{H}, \mathrm{d}, J$ 7.1, C $(\alpha) M e), 1.42(9 \mathrm{H}, \mathrm{s}, \mathrm{CMe}), 3.03(1 \mathrm{H}, \mathrm{d}, J$ 6.6, $\mathrm{OH}), 3.49-3.54(1 \mathrm{H}, \mathrm{m}, \mathrm{C}(3) H), 3.80\left(1 \mathrm{H}, \mathrm{d}, J\right.$ 15.0, $\left.\mathrm{NCH}_{\mathrm{A}} \mathrm{H}_{\mathrm{B}} \mathrm{Ph}\right), 3.84-3.89\left(2 \mathrm{H}, \mathrm{m}, \mathrm{C}(4) H_{2}\right), 3.99$ $(1 \mathrm{H}, \mathrm{dd}, J 6.6,1.7, \mathrm{C}(2) H), 4.02(1 \mathrm{H}, \mathrm{q}, J 7.1, \mathrm{C}(\alpha) H), 4.20\left(1 \mathrm{H}, \mathrm{d}, J 15.0, \mathrm{NCH}_{\mathrm{A}} H_{\mathrm{B}} \mathrm{Ph}\right), 7.21-7.47(10 \mathrm{H}, \mathrm{m}$, $P h)$. 


\section{4. tert-Butyl $(R, R, R)-2$-benzyloxy-3-[N-benzyl- $N$-( $\alpha$-methylbenzyl)amino]-4-}

\section{(triisopropylsilyloxy)butanoate 8}

A solution of $7(13.1 \mathrm{~g}, 24.2 \mathrm{mmol},>95: 5 \mathrm{dr})$ in THF $(290 \mathrm{~mL})$ was added to a stirred solution of $\mathrm{NaH}(60 \%$ in mineral oil, $1.93 \mathrm{~g}, 48.4 \mathrm{mmol})$ in $\mathrm{THF}(290 \mathrm{~mL})$ at $0{ }^{\circ} \mathrm{C}$ and the resultant mixture was stirred at $0{ }^{\circ} \mathrm{C}$ for $1 \mathrm{~h} . \mathrm{BnBr}(5.76 \mathrm{~mL}, 48.4 \mathrm{mmol})$ was added and the resultant mixture was allowed to warm to $\mathrm{rt}$ over $18 \mathrm{~h}$ then satd aq $\mathrm{NH}_{4} \mathrm{Cl}(50 \mathrm{~mL})$ and $\mathrm{Et}_{2} \mathrm{O}(300 \mathrm{~mL})$ were added. The resultant mixture was washed with brine $(300 \mathrm{~mL})$ and the aqueous layers were extracted with $\mathrm{Et}_{2} \mathrm{O}(3 \times 200 \mathrm{~mL})$ then the combined organic layers were dried and concentrated in vacuo. Purification via flash column chromatography (eluent $30-40{ }^{\circ} \mathrm{C}$ petroleum ether/ $\operatorname{Et}_{2} \mathrm{O}, 1: 0$ to $\left.30: 1\right)$ gave 8 as a colourless viscous oil $(14.0 \mathrm{~g}, 92 \%,>95: 5 \mathrm{dr}) ;^{13}[\alpha]_{\mathrm{D}}^{25}+24.1$ (c 1.0 in $\left.\mathrm{CHCl}_{3}\right)$; $\left\{\right.$ lit. $^{13}$ for ent-8 $[\alpha]_{\mathrm{D}}^{25}-33.1$ (c 0.7 in $\left.\mathrm{CHCl}_{3}\right) ; \delta_{\mathrm{H}}\left(400 \mathrm{MHz}, \mathrm{CDCl}_{3}\right) 1.04(21 \mathrm{H}, \mathrm{m}$, $\left.\mathrm{Si}(\mathrm{CHMe})_{3}\right), 1.35(3 \mathrm{H}, \mathrm{d}, J$ 6.9, C( $(\alpha) \mathrm{Me}), 1.42(9 \mathrm{H}, \mathrm{s}, \mathrm{CMe}), 3.48-3.53(1 \mathrm{H}, \mathrm{m}, \mathrm{C}(3) H), 3.65(1 \mathrm{H}, \mathrm{d}, J$ 15.2, $\left.\mathrm{NCH}_{\mathrm{A}} \mathrm{H}_{\mathrm{B}} \mathrm{Ph}\right), 3.75(1 \mathrm{H}, \mathrm{d}, J 2.7, \mathrm{C}(2) H), 3.83\left(1 \mathrm{H}, \mathrm{dd}, J 11.0,3.7, \mathrm{C}(4) H_{\mathrm{A}}\right), 3.94-4.06(2 \mathrm{H}, \mathrm{m}$, $\left.\mathrm{C}(4) H_{\mathrm{B}}, \mathrm{C}(\alpha) H\right), 4.18\left(1 \mathrm{H}, \mathrm{d}, J 11.2, \mathrm{OCH}_{\mathrm{A}} \mathrm{H}_{\mathrm{B}} \mathrm{Ph}\right), 4.26\left(1 \mathrm{H}, \mathrm{d}, J 15.2, \mathrm{NCH}_{\mathrm{A}} H_{\mathrm{B}} \mathrm{Ph}\right), 4.54(1 \mathrm{H}, \mathrm{d}, J 11.2$, $\left.\mathrm{OCH}_{\mathrm{A}} H_{\mathrm{B}} \mathrm{Ph}\right), 7.17-7.31(11 \mathrm{H}, \mathrm{m}, P h), 7.36(2 \mathrm{H}, \mathrm{d}, J 7.1, P h), 7.44(2 \mathrm{H}, \mathrm{d}, J 7.3, P h)$.

\section{5. $(R, R, R)-2-B e n z y l o x y-3-[N$-benzyl- $N$-( $\alpha$-methylbenzyl)amino-4-(triisopropylsilyloxy)butan-1-ol 9}

Method A: DIBAL-H (1.0 M in $\left.\mathrm{CH}_{2} \mathrm{Cl}_{2}, 29.9 \mathrm{~mL}, 29.9 \mathrm{mmol}\right)$ was added to a stirred solution of 8 (4.72 $\mathrm{g}$, $7.47 \mathrm{mmol},>95: 5 \mathrm{dr})$ in $\mathrm{CH}_{2} \mathrm{Cl}_{2}(125 \mathrm{~mL})$ at $-78{ }^{\circ} \mathrm{C}$ then stirred at $\mathrm{rt}$ for $2 \mathrm{~h}$. Satd aq $\mathrm{NH}_{4} \mathrm{Cl}(30 \mathrm{~mL})$ was added and the resultant mixture was filtered through Celite ${ }^{\circledR}\left(\right.$ eluent $\mathrm{CH}_{2} \mathrm{Cl}_{2}$ ) then dried and concentrated in vacuo. Purification via flash column chromatography (eluent $30-40{ }^{\circ} \mathrm{C}$ petroleum ether/EtOAc, 15:1) gave 9 as a pale yellow viscous oil $(2.78 \mathrm{~g}, 68 \%,>95: 5 \mathrm{dr}) ;^{13}[\alpha]_{\mathrm{D}}^{25}+18.1$ (c 1.0 in $\left.\mathrm{CHCl}_{3}\right)$; $\left\{\right.$ lit. ${ }^{13}$ for ent-9: $[\alpha]_{\mathrm{D}}^{20}-9.5\left(\right.$ c 4.0 in $\left.\left.\mathrm{CHCl}_{3}\right)\right\} ; \delta_{\mathrm{H}}\left(400 \mathrm{MHz}, \mathrm{CDCl}_{3}\right)$ 1.05-1.18 (21H, m, Si(CHMe $\left.)_{3}\right), 1.43(3 \mathrm{H}, \mathrm{d}, J$ 6.9, $\mathrm{C}(\alpha) M e), 3.03(1 \mathrm{H}$, br s, $\mathrm{OH}), 3.18(1 \mathrm{H}, \mathrm{q}, J 5.7, \mathrm{C}(3) H), 3.41-3.50\left(3 \mathrm{H}, \mathrm{m}, \mathrm{C}(1) H_{2}, \mathrm{C}(2) H\right), 3.94-4.09(4 \mathrm{H}$, m, C(4) $\left.H_{\mathrm{A}}, \mathrm{C}(\alpha) H, \mathrm{NCH}_{2} \mathrm{Ph}\right), 4.16\left(1 \mathrm{H}, \mathrm{dd}, J 10.2,5.2, \mathrm{C}(4) H_{\mathrm{B}}\right), 4.36\left(1 \mathrm{H}, \mathrm{d}, J 11.5, \mathrm{OCH}_{\mathrm{A}} H_{\mathrm{B}} \mathrm{Ph}\right), 4.55$ $\left(1 \mathrm{H}, \mathrm{d}, J 11.5, \mathrm{OCH}_{\mathrm{A}} H_{\mathrm{B}} \mathrm{Ph}\right), 7.22-7.33(15 \mathrm{H}, \mathrm{m}, P h)$.

Method B - Step 1: A solution of $7(15.0 \mathrm{~g}, 27.6 \mathrm{mmol},>95: 5 \mathrm{dr})$ in THF $(250 \mathrm{~mL})$ was added to a stirred solution of $\mathrm{NaH}(60 \%$ in mineral oil, $2.21 \mathrm{~g}, 55.2 \mathrm{mmol})$ in THF $(250 \mathrm{~mL})$ at $0{ }^{\circ} \mathrm{C}$ and the resultant mixture was stirred at $0{ }^{\circ} \mathrm{C}$ for $1 \mathrm{~h}$. $\mathrm{BnBr}(6.56 \mathrm{~mL}, 55.2 \mathrm{mmol})$ was added and the resultant mixture was allowed to warm to rt over $18 \mathrm{~h}$ then satd aq $\mathrm{NH}_{4} \mathrm{Cl}(50 \mathrm{~mL})$ and $\mathrm{Et}_{2} \mathrm{O}(300 \mathrm{~mL})$ were added. The resultant mixture was washed with brine $(300 \mathrm{~mL})$ and the aqueous layers were extracted with $\mathrm{Et}_{2} \mathrm{O}(3 \times 200 \mathrm{~mL})$ then the combined organic layers were dried and concentrated in vacuo to give $\mathbf{8}$. 
Method B - Step 2: DIBAL-H (1.0 M in $\left.\mathrm{CH}_{2} \mathrm{Cl}_{2}, 110 \mathrm{~mL}, 110 \mathrm{mmol}\right)$ was added to a stirred solution of the residue of 8 from the previous step in $\mathrm{CH}_{2} \mathrm{Cl}_{2}(500 \mathrm{~mL})$ at $-78{ }^{\circ} \mathrm{C}$ then stirred at $\mathrm{rt}$ for $2 \mathrm{~h}$. Satd aq $\mathrm{NH}_{4} \mathrm{Cl}$ $(30 \mathrm{~mL})$ and satd aq Rochelle salt $(100 \mathrm{~mL})$ were added at $0{ }^{\circ} \mathrm{C}$ and the resultant mixture was allowed to warm to rt over $18 \mathrm{~h}$, then filtered through Celite ${ }^{\circledR}$ (eluent $\mathrm{CH}_{2} \mathrm{Cl}_{2}$ ), dried and concentrated in vacuo. Purification via flash column chromatography (eluent 30-40 ${ }^{\circ} \mathrm{C}$ petroleum ether/EtOAc, 15:1) gave 9 as a pale yellow viscous oil (13.4 g, 87\% from 7, >95:5 dr).

\subsection{Ethyl $(R, R, R, R)-3$-hydroxy-4-benzyloxy-5-[N-benzyl- $N$-( $(\alpha$-methylbenzyl)amino]-6-}

\section{(triisoproylsilyloxy)hexanoate 11 and ethyl $(3 S, 4 R, 5 R, \alpha R)-3-h y d r o x y-4-b e n z y l o x y-5-[N-$ benzyl- $N-(\alpha-$ methylbenzyl)amino]-6-(triisoproylsilyloxy)hexanoate 12}

Step 1: DMSO $(0.76 \mathrm{~mL}, 10.7 \mathrm{mmol})$ was added to a stirred solution of $(\mathrm{COCl})_{2}(0.45 \mathrm{~mL}, 5.34 \mathrm{mmol})$ in $\mathrm{CH}_{2} \mathrm{Cl}_{2}(15 \mathrm{~mL})$ at $-78{ }^{\circ} \mathrm{C}$ and the resultant mixture was allowed to stir at $-78{ }^{\circ} \mathrm{C}$ for $10 \mathrm{~min}$ then $9(1.50 \mathrm{~g}$, $2.67 \mathrm{mmol},>95: 5 \mathrm{dr})$ in $\mathrm{CH}_{2} \mathrm{Cl}_{2}(15 \mathrm{~mL})$ was added at $-78{ }^{\circ} \mathrm{C}$. The resultant mixture was allowed to stir at $-78{ }^{\circ} \mathrm{C}$ for $1 \mathrm{~h}$ then $\mathrm{Et}_{3} \mathrm{~N}(2.23 \mathrm{~mL}, 16.0 \mathrm{mmol})$ was added and the resultant mixture was allowed to stir at $-78{ }^{\circ} \mathrm{C}$ for $20 \mathrm{~min}$ then at $\mathrm{rt}$ for $20 \mathrm{~min}$. The organic layer was washed with $\mathrm{H}_{2} \mathrm{O}(2 \times 15 \mathrm{~mL})$ then the combined aqueous layers were extracted with $\mathrm{CH}_{2} \mathrm{Cl}_{2}(2 \times 15 \mathrm{~mL})$ then the combined organic layers were dried and concentrated in vacuo to give $\mathbf{1 0}$ as a pale yellow viscous oil; $[\alpha]_{\mathrm{D}}^{25}+30.2\left(\right.$ c 1.0 in $\left.\mathrm{CHCl}_{3}\right) ; v_{\max }$ (ATR) $2866(\mathrm{C}-\mathrm{H}), 1728(\mathrm{C}=\mathrm{O}) ; \delta_{\mathrm{H}}\left(400 \mathrm{MHz}, \mathrm{CDCl}_{3}\right)$ 1.01-1.10 (21H, m, Si(CHMe $\left.)_{3}\right), 1.38(3 \mathrm{H}, \mathrm{d}, J$ 7.0, $\mathrm{C}(\alpha) M e), 3.46-3.52(1 \mathrm{H}, \mathrm{m}, \mathrm{C}(3) H), 3.55(1 \mathrm{H}, \mathrm{dd}, J 4.2,2.5, \mathrm{C}(2) H), 3.77\left(1 \mathrm{H}, \mathrm{d}, J 14.7, \mathrm{NCH}_{\mathrm{A}} \mathrm{H}_{\mathrm{B}} \mathrm{Ph}\right)$, 3.81-3.91 (2H, m, C(4)H $\left.H_{\mathrm{A}}, \mathrm{C}(\alpha) H\right), 4.06\left(1 \mathrm{H}, \mathrm{dd}, J\right.$ 9.6, 7.6, C(4)H $\left.H_{\mathrm{B}}\right), 4.12\left(1 \mathrm{H}, \mathrm{d}, J 14.7, \mathrm{NCH}_{\mathrm{A}} H_{\mathrm{B}} \mathrm{Ph}\right), 4.34$ $\left(1 \mathrm{H}, \mathrm{d}, J 11.3, \mathrm{OCH}_{\mathrm{A}} \mathrm{H}_{\mathrm{B}} \mathrm{Ph}\right), 4.48\left(1 \mathrm{H}, \mathrm{d}, J 11.3, \mathrm{OCH}_{\mathrm{A}} H_{\mathrm{B}} \mathrm{Ph}\right), 7.20-7.38(15 \mathrm{H}, \mathrm{m}, P h), 9.19(1 \mathrm{H}, \mathrm{d}, J 2.5$, $\mathrm{C}(1) H) ; \delta_{\mathrm{C}}\left(100 \mathrm{MHz}, \mathrm{CDCl}_{3}\right) 11.9\left(\mathrm{Si}\left(\mathrm{CHMe}_{2}\right)_{3}\right), 17.1(\mathrm{C}(\alpha) M e), 18.0\left(\mathrm{Si}(\mathrm{CHMe})_{3}\right), 51.9\left(\mathrm{NCH}_{2} \mathrm{Ph}\right), 57.1$ $(C(\alpha)), 59.6(C(3)), 62.0(C(4)), 72.6\left(\mathrm{OCH}_{2} \mathrm{Ph}\right), 83.2(C(2)), 126.7,127.1,127.8,128.1,128.2,128.2,128.3$, 128.3, $128.4(o, m, p-P h), 137.2,141.2,142.2(i-P h), 202.9(C(1)) ; m / z\left(\mathrm{ESI}^{+}\right) 592\left(\left[\mathrm{M}^{+} \mathrm{CH}_{5} \mathrm{O}\right]^{+}, 100 \%\right)$; HRMS $\left(\mathrm{ESI}^{+}\right) \mathrm{C}_{36} \mathrm{H}_{54} \mathrm{NO}_{4} \mathrm{Si}^{+}\left(\left[\mathrm{M}+\mathrm{CH}_{5} \mathrm{O}\right]^{+}\right)$requires 592.3817; found 592.3807.

Step 2: BuLi (2.2 M in hexanes, $1.70 \mathrm{~mL}, 3.74 \mathrm{mmol})$ was added to a stirred solution of ${ }^{\mathrm{i}} \mathrm{Pr}_{2} \mathrm{NH}(0.52 \mathrm{~mL}$, $3.74 \mathrm{mmol})$ in THF $(5 \mathrm{~mL})$ at $-78^{\circ} \mathrm{C}$ and the resultant mixture was stirred at $-78{ }^{\circ} \mathrm{C}$ for $20 \mathrm{~min}$ then EtOAc $(0.37 \mathrm{~mL}, 3.74 \mathrm{mmol})$ was added at $-78{ }^{\circ} \mathrm{C}$ and the resultant mixture was stirred at $-78{ }^{\circ} \mathrm{C}$ for $30 \mathrm{~min}$. A solution of the residue of $\mathbf{1 0}$ from the previous step in THF $(10 \mathrm{~mL})$ was added at $-78{ }^{\circ} \mathrm{C}$ and the resultant mixture was allowed to stir at $-78{ }^{\circ} \mathrm{C}$ for $1 \mathrm{~h}$ then at $0{ }^{\circ} \mathrm{C}$ for $2 \mathrm{~h}$ before satd aq $\mathrm{NH}_{4} \mathrm{Cl}(2 \mathrm{~mL})$ and $\mathrm{Et}_{2} \mathrm{O}(10$ $\mathrm{mL}$ ) was added. The aqueous layer was extracted with $\mathrm{Et}_{2} \mathrm{O}(3 \times 10 \mathrm{~mL})$ then the combined organic layers 
were washed with brine $(25 \mathrm{~mL})$ then were dried and concentrated in vacuo to give a $\sim 65: 35$ mixture of 11 and 12, respectively. Purification via flash column chromatography (eluent $30-40{ }^{\circ} \mathrm{C}$ petroleum ether/EtOAc, 10:1) gave 12 as a pale yellow viscous oil (566 mg, 33\% from 9, >95:5 dr); $[\alpha]_{\mathrm{D}}^{25}+8.8(c 1.0$ in $\left.\mathrm{CHCl}_{3}\right) ; v_{\max }(\mathrm{ATR}) 3519$ (broad, O-H), $1731(\mathrm{C}=\mathrm{O}) ; \delta_{\mathrm{H}}\left(500 \mathrm{MHz}, \mathrm{CDCl}_{3}\right)$ 1.08-1.20 (21H, m, $\left.\mathrm{Si}\left(\mathrm{CHMe}_{2}\right)_{3}\right), 1.28\left(3 \mathrm{H}, \mathrm{t}, J\right.$ 7.2, $\left.\mathrm{OCH}_{2} \mathrm{CH}_{3}\right), 1.48(3 \mathrm{H}, \mathrm{d}, J 6.9, \mathrm{C}(\alpha) \mathrm{Me}), 1.79\left(1 \mathrm{H}, \mathrm{dd}, J 16.3,3.6, \mathrm{C}(2) \mathrm{H}_{\mathrm{A}}\right)$, $2.22\left(1 \mathrm{H}, \mathrm{dd}, J 16.3,8.9, \mathrm{C}(2) H_{\mathrm{B}}\right), 3.04-3.08(1 \mathrm{H}, \mathrm{m}, \mathrm{C}(5) H), 3.61(1 \mathrm{H}, \mathrm{dd}, J 7.2,3.4, \mathrm{C}(4) H), 3.99(1 \mathrm{H}, \mathrm{d}, J$ 14.1, $\left.\mathrm{NCH}_{\mathrm{A}} \mathrm{H}_{\mathrm{B}} \mathrm{Ph}\right), 4.03\left(1 \mathrm{H}, \mathrm{d}, J 14.1, \mathrm{NCH}_{\mathrm{A}} H_{\mathrm{B}} \mathrm{Ph}\right), 4.06\left(1 \mathrm{H}, \mathrm{dd}, J 10.1,6.6, \mathrm{C}(6) H_{\mathrm{A}}\right), 4.09-4.16(3 \mathrm{H}, \mathrm{m}$, $\left.\mathrm{OCH}_{2} \mathrm{CH}_{3}, \mathrm{C}(\alpha) H\right), 4.18-4.23\left(2 \mathrm{H}, \mathrm{m}, \mathrm{C}(6) H_{\mathrm{B}}, \mathrm{OH}\right), 4.28-4.33(1 \mathrm{H}, \mathrm{m}, \mathrm{C}(3) H), 4.48(1 \mathrm{H}, \mathrm{d}, J 11.5$, $\left.\mathrm{OCH}_{\mathrm{A}} \mathrm{H}_{\mathrm{B}} \mathrm{Ph}\right), 4.76\left(1 \mathrm{H}, \mathrm{d}, J 11.5, \mathrm{OCH}_{\mathrm{A}} H_{\mathrm{B}} \mathrm{Ph}\right), 7.21-7.38(15 \mathrm{H}, \mathrm{m}, P h) ; \delta_{\mathrm{C}}\left(125 \mathrm{MHz}, \mathrm{CDCl}_{3}\right) 12.0$ $\left(\mathrm{Si}\left(\mathrm{CHMe}_{2}\right)_{3}\right), 14.1\left(\mathrm{OCH}_{2} \mathrm{CH}_{3}\right), 17.1(\mathrm{C}(\alpha) M e), 18.1\left(\mathrm{Si}(\mathrm{CHMe})_{3}\right), 36.4(C(2)), 51.8\left(\mathrm{NCH}_{2} \mathrm{Ph}\right), 57.7$ $(C(\alpha)), 59.0(C(5)), 60.3\left(\mathrm{OCH}_{2} \mathrm{CH}_{3}\right), 62.5(C(6)), 69.3(C(3)), 73.0\left(\mathrm{OCH}_{2} \mathrm{Ph}\right), 81.3(C(4)), 126.7,126.8$, 127.3, 127.5, 127.9, 128.1, 128.2, 128.2, 129.0 (o,m,p-Ph), 138.5, 140.9, $144.6(i-P h), 173.0(C(1)) ; \mathrm{m} / z$ $\left(\mathrm{ESI}^{+}\right) 648\left([\mathrm{M}+\mathrm{H}]^{+}, 100 \%\right)$; HRMS $\left(\mathrm{ESI}^{+}\right) \mathrm{C}_{39} \mathrm{H}_{58} \mathrm{NO}_{5} \mathrm{Si}^{+}\left([\mathrm{M}+\mathrm{H}]^{+}\right)$requires 648.4079; found 648.4062. Further elution gave a 74:26 mixture of $\mathbf{1 1}$ and 12, respectively, as a pale yellow viscous oil (210 mg, 12\% from 9). Further elution gave 11 as a pale yellow viscous oil $\left(675 \mathrm{mg}, 39 \%\right.$ from 9, >95:5 dr); $[\alpha]_{\mathrm{D}}^{25}+13.1(c$ 1.0 in $\left.\mathrm{CHCl}_{3}\right) ; v_{\max }(\mathrm{ATR}) 3505(\mathrm{O}-\mathrm{H}), 1735(\mathrm{C}=\mathrm{O}) ; \delta_{\mathrm{H}}\left(500 \mathrm{MHz}, \mathrm{CDCl}_{3}\right)$ 1.08-1.18 $(21 \mathrm{H}, \mathrm{m}$, $\left.\mathrm{Si}\left(\mathrm{CHMe}_{2}\right)_{3}\right), 1.28\left(3 \mathrm{H}, \mathrm{t}, J\right.$ 14.3, $\left.\mathrm{OCH}_{2} \mathrm{CH}_{3}\right), 1.50(3 \mathrm{H}, \mathrm{d}, J$ 6.9, $\mathrm{C}(\alpha) M e), 1.89(1 \mathrm{H}, \mathrm{dd}, J$ 15.4, 9.5, $\left.\mathrm{C}(2) H_{\mathrm{A}}\right), 2.08\left(1 \mathrm{H}, \mathrm{dd}, J 15.4,3.9, \mathrm{C}(2) H_{\mathrm{B}}\right), 3.36-3.43(2 \mathrm{H}, \mathrm{m}, \mathrm{C}(4) H, \mathrm{C}(5) H), 3.72(1 \mathrm{H}, \mathrm{br}$ s, OH), 4.01 $\left(1 \mathrm{H}, \mathrm{d}, J\right.$ 14.3, $\left.\mathrm{NCH}_{\mathrm{A}} \mathrm{H}_{\mathrm{B}} \mathrm{Ph}\right), 4.09\left(1 \mathrm{H}, \mathrm{d}, J 14.3, \mathrm{NCH}_{\mathrm{A}} H_{\mathrm{B}} \mathrm{Ph}\right), 4.10-4.18\left(5 \mathrm{H}, \mathrm{m}, \mathrm{C}(6) H_{2}, \mathrm{C}(\alpha) H\right.$, $\left.\mathrm{OCH}_{2} \mathrm{CH}_{3}\right), 4.43\left(1 \mathrm{H}, \mathrm{d}, J 11.4, \mathrm{OCH}_{\mathrm{A}} H_{\mathrm{B}} \mathrm{Ph}\right), 4.44-4.48(1 \mathrm{H}$, br s, $\mathrm{C}(3) H), 4.51(1 \mathrm{H}, \mathrm{d}, J 11.4$, $\left.\mathrm{OCH}_{\mathrm{A}} H_{\mathrm{B}} \mathrm{Ph}\right), 7.20-7.38(15 \mathrm{H}, \mathrm{m}, \mathrm{Ph}) ; \delta_{\mathrm{C}}\left(125 \mathrm{MHz}, \mathrm{CDCl}_{3}\right) 12.1\left(\mathrm{Si}\left(\mathrm{CHMe}_{2}\right)_{3}\right), 14.2\left(\mathrm{OCH}_{2} \mathrm{CH}_{3}\right), 17.6$ $(\mathrm{C}(\alpha) M e), 18.2\left(\mathrm{Si}(\mathrm{CHMe})_{3}\right), 38.3(C(2)), 52.2\left(\mathrm{NCH}_{2} \mathrm{Ph}\right), 58.7(C(5)), 59.0(C(\alpha)), 60.2\left(\mathrm{OCH}_{2} \mathrm{CH}_{3}\right), 62.7$ $(C(6)), 68.4(C(3)), 73.6\left(\mathrm{OCH}_{2} \mathrm{Ph}\right), 78.9(C(4)), 127.0,127.1,127.5,127.6,128.1,128.2,128.4,128.4$, $129.1(o, m, p-P h), 138.0,140.7,144.6(i-P h), 172.1(C(1)) ; m / z\left(\mathrm{ESI}^{+}\right) 648\left([\mathrm{M}+\mathrm{H}]^{+}, 100 \%\right) ; \mathrm{HRMS}\left(\mathrm{ESI}^{+}\right)$ $\mathrm{C}_{39} \mathrm{H}_{58} \mathrm{NO}_{5} \mathrm{Si}^{+}\left([\mathrm{M}+\mathrm{H}]^{+}\right)$requires 648.4079; found 648.4070.

\section{7. $(R, R, R, R)-4-B e n z y l o x y-5-[N$-benzyl- $N$-( $\alpha$-methylbenzyl)amino]-6-(triisopropylsilyoxy)hexa-1,3-}

\section{diol 13}

Method $A:{ }^{30} \mathrm{BH}_{3} \cdot \mathrm{SMe}_{2}(2.0 \mathrm{M}$ in THF, $0.26 \mathrm{~mL}, 0.527 \mathrm{mmol})$ was added to a stirred solution of $14(100 \mathrm{mg}$, $0.170 \mathrm{mmol},>95: 5 \mathrm{dr})$ in THF $(1.6 \mathrm{~mL})$ at $0{ }^{\circ} \mathrm{C}$ and the resultant mixture was stirred at $\mathrm{rt}$ for $2 \mathrm{~h}$ then cooled to $0{ }^{\circ} \mathrm{C}$ and $\mathrm{MeOH} / \mathrm{THF}(1: 1,1.8 \mathrm{~mL}), 3 \mathrm{M}$ aq $\mathrm{NaOH}(1.73 \mathrm{~mL})$ and $30 \% \mathrm{aq}_{2} \mathrm{O}_{2}(0.58 \mathrm{~mL}, 5.10 \mathrm{mmol})$ 
were added sequentially and the resultant mixture was left to stir at $\mathrm{rt}$ for $2 \mathrm{~h}$. EtOAc $(5 \mathrm{~mL})$ and $\mathrm{H}_{2} \mathrm{O}(5 \mathrm{~mL})$ were added and the aqueous layer was extracted with EtOAc $(3 \times 5 \mathrm{~mL})$ then the combined organic layers were dried and concentrated in vacuo. Purification via flash column chromatography (eluent $30-40{ }^{\circ} \mathrm{C}$ petroleum ether/EtOAc, 15:1 increased to 2:1) gave $\mathbf{1 3}$ as a colourless viscous oil (36 mg, 35\%, >95:5 dr); $[\alpha]_{\mathrm{D}}^{25}+19.1\left(c \quad 1.0\right.$ in $\left.\mathrm{CHCl}_{3}\right) ; v_{\max }(\mathrm{ATR}) 3407(\mathrm{O}-\mathrm{H}) ; \delta_{\mathrm{H}}\left(500 \mathrm{MHz}, \mathrm{CDCl}_{3}\right)$ 1.08-1.15 (21H, m,

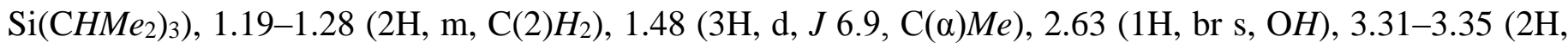
m, C(4)H, C(5)H), 3.50-3.65 (2H, m, C(1)H $), 3.76\left(1 \mathrm{H}\right.$, br s, OH), 3.97-4.16 (6H, m, C(3)H, C(6) $H_{2}$, $\left.\mathrm{C}(\alpha) H, \mathrm{NCH}_{2} \mathrm{Ph}\right), 4.46\left(1 \mathrm{H}, \mathrm{d}, J 11.4, \mathrm{OCH}_{\mathrm{A}} \mathrm{H}_{\mathrm{B}} \mathrm{Ph}\right), 4.52\left(1 \mathrm{H}, \mathrm{d}, J 11.4, \mathrm{OCH}_{\mathrm{A}} H_{\mathrm{B}} \mathrm{Ph}\right), 7.21-7.35(15 \mathrm{H}, \mathrm{m}$, $P h) ; \delta_{\mathrm{C}}\left(125 \mathrm{MHz}, \mathrm{CDCl}_{3}\right) 12.0\left(\mathrm{Si}\left(\mathrm{CHMe}_{2}\right)_{3}\right), 17.5(\mathrm{C}(\alpha) M e), 18.1\left(\mathrm{Si}(\mathrm{CHMe})_{3}\right), 34.7(C(2)), 52.1$ $\left(\mathrm{NCH}_{2} \mathrm{Ph}\right), 58.7(C(\alpha)), 58.9(C(5)), 62.1(C(1)), 62.7(C(6)), 72.0(C(3)), 73.9\left(\mathrm{OCH}_{2} \mathrm{Ph}\right), 80.3(C(4))$, 127.0, 127.0, 127.6, 128.0, 128.3, 128.4, $129.1(o, m, p-P h), 138.1,140.7,144.5(i-P h) ; m / z\left(\mathrm{ESI}^{+}\right) 606$ $\left([\mathrm{M}+\mathrm{H}]^{+}, 100 \%\right) ; \mathrm{HRMS}\left(\mathrm{ESI}^{+}\right) \mathrm{C}_{37} \mathrm{H}_{56} \mathrm{NO}_{4} \mathrm{Si}^{+}\left([\mathrm{M}+\mathrm{H}]^{+}\right)$requires 606.3973; found 606.3972.

Method B: $\mathrm{LiAlH}_{4}$ was added to a stirred solution of $\mathbf{1 1}(100 \mathrm{mg}, 0.154 \mathrm{mmol},>95: 5 \mathrm{dr})$ in $\mathrm{THF}$ at $0{ }^{\circ} \mathrm{C}$ and allowed to warm to rt over $18 \mathrm{~h}$ then $2.0 \mathrm{M}$ aq NaOH $(2 \mathrm{~mL})$ was added and the resultant mixture was stirred at $\mathrm{rt}$ for $2 \mathrm{~h}$ then filtered and concentrated in vacuo. Purification via flash column chromatography (eluent 40-60 ${ }^{\circ} \mathrm{C}$ petroleum ether/EtOAc/35\% aq $\left.\mathrm{NH}_{4} \mathrm{OH}, 66: 33: 1\right)$ gave $\mathbf{1 3}$ as a colourless viscous oil (61 mg, $65 \%,>95: 5 \mathrm{dr})$.

\section{8. $(R, R, R, R)-1$-(Triisopropylsilyloxy)-2-[ $N$-benzyl- $N$-( $(\alpha$-methylbenzyl)amino]-3-benzyloxyhex-5-en-4- ol 14}

Vinylmagnesium bromide (1.0 M in THF, $0.54 \mathrm{~mL}, 0.54 \mathrm{mmol})$ was added to 10 (250 $\mathrm{mg}, 0.447 \mathrm{mmol}$, $>95: 5 \mathrm{dr})$ in $\mathrm{THF}(5 \mathrm{~mL})$ at $0{ }^{\circ} \mathrm{C}$ and allowed to warm to $\mathrm{rt}$ over $18 \mathrm{~h}$ then cooled to $0{ }^{\circ} \mathrm{C}$ and satd aq $\mathrm{NH}_{4} \mathrm{Cl}$ $(1 \mathrm{~mL})$ was added and the resultant mixture was concentrated in vacuo. The residue was partitioned between EtOAc $(10 \mathrm{~mL})$ and $\mathrm{H}_{2} \mathrm{O}(10 \mathrm{~mL})$ and the aqueous layer was extracted with EtOAc $(3 \times 10 \mathrm{~mL})$ then the combined organic layers were washed with brine $(25 \mathrm{~mL})$ then dried and concentrated in vacuo to give $\mathbf{1 4}$ in 70:30 dr. Purification via flash column chromatography (eluent $30-40{ }^{\circ} \mathrm{C}$ petroleum ether/EtOAc, 25:1) gave 14 as a colourless viscous oil $\left(133 \mathrm{mg}, 51 \%\right.$, >95:5 dr) $;^{12,17}[\alpha]_{\mathrm{D}}^{25}+38.2$ (c 1.0 in $\left.\mathrm{CHCl}_{3}\right) ;\left\{\right.$ lit. $^{12}$ $[\alpha]_{\mathrm{D}}^{20}+36.5\left(c 1.0\right.$ in $\left.\left.\mathrm{CHCl}_{3}\right)\right\} ; \delta_{\mathrm{H}}\left(400 \mathrm{MHz}, \mathrm{CDCl}_{3}\right)$ 1.06-1.15 (21H, m, Si(CHMe $\left.)_{3}\right), 1.39$ (3H, d, J 6.8, $\mathrm{C}(\alpha) M e), 3.18(1 \mathrm{H}, \mathrm{d}, J$ 6.9, $\mathrm{OH}), 3.22(1 \mathrm{H}, \mathrm{dd}, J$ 7.8, 2.4, C(3)H), $3.43(1 \mathrm{H}$, app dt, $J 7.8,4.8, \mathrm{C}(2) H), 3.96$ $\left(2 \mathrm{H}\right.$, app s, $\left.\mathrm{NCH}_{2} \mathrm{Ph}\right), 4.02\left(1 \mathrm{H}, \mathrm{dd}, J 10.4,7.8, \mathrm{C}(1) H_{\mathrm{A}}\right), 4.06-4.18\left(2 \mathrm{H}, \mathrm{m}, \mathrm{C}(1) H_{\mathrm{B}}, \mathrm{C}(\alpha) H\right), 4.29(1 \mathrm{H}, \mathrm{d}, J$ 11.3, $\left.\mathrm{OCH}_{\mathrm{A}} \mathrm{H}_{\mathrm{B}} \mathrm{Ph}\right), 4.46(1 \mathrm{H}$, br s, $\mathrm{C}(4) H), 4.51\left(1 \mathrm{H}, \mathrm{d}, J 11.3, \mathrm{OCH}_{\mathrm{A}} H_{\mathrm{B}} \mathrm{Ph}\right), 5.03(1 \mathrm{H}$, app dt, $J 10.5,1.8$, 
$\left.\mathrm{C}(6) H_{\mathrm{A}}\right), 5.23\left(1 \mathrm{H}\right.$, app dt, $\left.J 17.2,1.8, \mathrm{C}(6) H_{\mathrm{B}}\right), 5.58(1 \mathrm{H}, \mathrm{ddd}, J 17.2,10.5,4.7, \mathrm{C}(5) H), 7.15-7.41(15 \mathrm{H}, \mathrm{m}$, $P h)$.

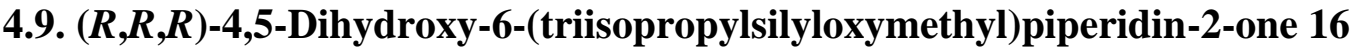

Step 1: $\mathrm{Pd}(\mathrm{OH})_{2} / \mathrm{C}(160 \mathrm{mg}, 20 \mathrm{~mol} \%)$ was added to a vigorously stirred solution of 11 (802 mg, $1.24 \mathrm{mmol}$, $>95: 5 \mathrm{dr})$ in degassed $\mathrm{MeOH}(10 \mathrm{~mL})$. The reaction mixture was stirred under $\mathrm{H}_{2}(1 \mathrm{~atm})$ for $24 \mathrm{~h}$ then filtered through Celite (eluent $\mathrm{MeOH}$ ) and concentrated in vacuo.

Step 2: $\mathrm{Na}(285 \mathrm{mg}, 12.4 \mathrm{mmol})$ was added to a stirred solution of naphthalene $(1.91 \mathrm{~g}, 14.9 \mathrm{mmol})$ in DME $(14 \mathrm{~mL})$ at $\mathrm{rt}$ and stirred at $\mathrm{rt}$ for $2 \mathrm{~h}$ then cooled to $-78^{\circ} \mathrm{C}$. The residue from the previous step was dissolved in DME (33 mL) and added at $-78{ }^{\circ} \mathrm{C}$ and the resultant mixture was allowed to warm to rt over $18 \mathrm{~h}$. Satd aq $\mathrm{NH}_{4} \mathrm{CL}(5 \mathrm{~mL})$ was added and the aqueous layer was extracted with $\mathrm{Et}_{2} \mathrm{O}(3 \times 25 \mathrm{~mL})$. The combined organic layers were washed with brine $(40 \mathrm{~mL})$ then dried and concentrated in vacuo. Purification via flash column chromatography (eluent $\mathrm{CHCl}_{3} /{ }^{\mathrm{i}} \mathrm{PrOH} / 35 \%$ aq $\mathrm{NH}_{4} \mathrm{OH}, 78: 12: 1$ ) to give $\mathbf{1 6}$ as an off-white solid $\left(220 \mathrm{mg}, 56 \%\right.$ from 11, >95:5 dr); mp $109-111^{\circ} \mathrm{C} ;[\alpha]_{\mathrm{D}}^{25}+16.1\left(\right.$ c 1.0 in $\left.\mathrm{CHCl}_{3}\right) ; v_{\max }$ (ATR) $3398(\mathrm{O}-\mathrm{H}$, $\mathrm{N}-\mathrm{H}), 1651(\mathrm{C}=\mathrm{O}) ; \delta_{\mathrm{H}}\left(500 \mathrm{MHz}, \mathrm{CDCl}_{3}, 18.9 \mathrm{mM}\right)^{31} 1.04-1.09\left(21 \mathrm{H}, \mathrm{m}, \mathrm{Si}(\mathrm{CHMe})_{3}\right), 2.37(1 \mathrm{H}, \mathrm{dd}$, $J$ 17.4, 11.0, C(3) $\left.H_{\mathrm{A}}\right), 2.68(1 \mathrm{H}, \mathrm{d}, J 3.5, \mathrm{OH}), 2.82\left(1 \mathrm{H}, \mathrm{dd}, J 17.4,5.7, \mathrm{C}(3) H_{\mathrm{B}}\right), 3.09(1 \mathrm{H}, \mathrm{d}, J 2.7, \mathrm{OH})$, $3.36(1 \mathrm{H}$, app td, $J 8.5,4.5, \mathrm{C}(6) H), 3.47(1 \mathrm{H}, \mathrm{td}, J 9.1,2.5, \mathrm{C}(5) H), 3.67\left(1 \mathrm{H}, \mathrm{dd}, J 9.7,8.2, \mathrm{C}\left(1^{\prime}\right) H_{\mathrm{A}}\right)$, 3.89-3.96 (1H, m, C(4)H), $4.04\left(1 \mathrm{H}, \mathrm{dd}, J\right.$ 9.7, 4.5, C(1')H $\left.H_{\mathrm{B}}\right), 5.95(1 \mathrm{H}$, br s, $\mathrm{NH}) ; \delta_{\mathrm{C}}\left(125 \mathrm{MHz}, \mathrm{CDCl}_{3}\right.$, $18.9 \mathrm{mM})^{31} 11.8\left(\mathrm{Si}\left(\mathrm{CHMe}_{2}\right)_{3}\right), 17.9\left(\mathrm{Si}(\mathrm{CHMe})_{3}\right), 37.8(C(3)), 56.3(C(6)), 65.6\left(C\left(1^{\prime}\right)\right), 69.0(C(4)), 72.5$ $(C(5)), 169.3(C(2)) ; \delta_{\mathrm{H}}\left(500 \mathrm{MHz}, \mathrm{CDCl}_{3}, 139 \mathrm{mM}\right)^{31} 1.00-1.16\left(21 \mathrm{H}, \mathrm{m}, \mathrm{Si}(\mathrm{CHMe})_{3}\right), 2.34(1 \mathrm{H}, \mathrm{dd}$, $\left.J 17.5,10.8, \mathrm{C}(3) H_{\mathrm{A}}\right), 2.78\left(1 \mathrm{H}, \mathrm{dd}, J 17.5,6.0, \mathrm{C}(3) H_{\mathrm{B}}\right), 3.31-3.36(1 \mathrm{H}, \mathrm{m}, \mathrm{C}(6) H), 3.42(1 \mathrm{H}, \mathrm{t}, J 9.0$, $\mathrm{C}(5) H), 3.63\left(1 \mathrm{H}, \mathrm{dd}, J 9.6,8.5, \mathrm{C}\left(1^{\prime}\right) H_{\mathrm{A}}\right), 3.83\left(1 \mathrm{H}, \mathrm{q}, J\right.$ 8.6, C(4)H), $4.06\left(1 \mathrm{H}, \mathrm{dd}, J 9.8,3.9, \mathrm{C}\left(1^{\prime}\right) H_{\mathrm{B}}\right), 6.25$ $(1 \mathrm{H}$, br s, $\mathrm{NH}) ; \delta_{\mathrm{C}}\left(125 \mathrm{MHz}, \mathrm{CDCl}_{3}, 139 \mathrm{mM}\right)^{31} 11.7\left(\mathrm{Si}\left(\mathrm{CHMe}_{2}\right)_{3}\right), 17.9\left(\mathrm{Si}(\mathrm{CHMe})_{3}\right), 37.7(C(3)), 56.7$ $(C(6)), 65.2\left(C\left(1^{\prime}\right)\right), 68.8(C(4)), 71.9(C(5)), 170.3(C(2)) ; \mathrm{m} / z\left(\mathrm{ESI}^{+}\right) 318\left([\mathrm{M}+\mathrm{H}]^{+}, 100 \%\right), 340\left([\mathrm{M}+\mathrm{Na}]^{+}\right.$, 63\%); HRMS $\left(\mathrm{ESI}^{+}\right) \mathrm{C}_{15} \mathrm{H}_{32} \mathrm{NO}_{4} \mathrm{Si}^{+}\left([\mathrm{M}+\mathrm{H}]^{+}\right)$requires 318.2095; found 318.2096.

\subsubsection{X-ray crystal structure determination for 16}

Data were collected using an Oxford Diffraction SuperNova diffractometer with graphite monochromated $\mathrm{Cu}-\mathrm{K} \alpha$ radiation using standard procedures at $250 \mathrm{~K}$. The structures were solved by direct methods (SIR92); all non-hydrogen atoms were refined with anisotropic thermal parameters. Hydrogen atoms were added at idealised positions. The structures were refined using CRYSTALS. ${ }^{18,32}$ 
X-ray crystal structure data for $16\left[\mathrm{C}_{15} \mathrm{H}_{31} \mathrm{NO}_{4} \mathrm{Si}\right]: M=317.50$, monoclinic, space group $P 2{ }_{1}$, $a=14.0713(7) \AA, b=8.2150(4) \AA, c=16.8488(8) \AA, \beta=107.330(5)^{\circ}, V=1859.23(17) \AA^{3}, Z=4, \mu=1.232$ $\mathrm{mm}^{-1}$, colourless prism, crystal dimensions $=0.12 \times 0.13 \times 0.25 \mathrm{~mm}^{3}$. A total of 7686 unique reflections were measured for $4<\theta<76$ and 7655 reflections were used in the refinement. The final parameters were $w R_{2}=0.257$ and $R_{1}=0.096[I>-3.0 \sigma(I)]$, with Flack enantiopole $=-0.02(6) .{ }^{19}$

\subsection{1,2,5-Trideoxy-1,5-imino-D-arabino-hexitol [D-fagomine] 1}

$\mathrm{BH}_{3} \cdot \mathrm{SMe}_{2}(2.0 \mathrm{M}$ in THF, $1.10 \mathrm{~mL}, 2.20 \mathrm{mmol})$ was added to a solution of $\mathbf{1 6}(100 \mathrm{mg}, 0.315 \mathrm{mmol},>95: 5$ dr) in THF $(6 \mathrm{~mL})$ at $0{ }^{\circ} \mathrm{C}$ and stirred at $0{ }^{\circ} \mathrm{C}$ for 30 min then warmed to $\mathrm{rt}$ over $2 \mathrm{~h}$ then $3 \mathrm{M}$ aq $\mathrm{NaOH}$ ( $2 \mathrm{~mL}$ ) and $30 \%$ aq $\mathrm{H}_{2} \mathrm{O}_{2}(2 \mathrm{~mL})$ were added and the resultant mixture was heated at $85{ }^{\circ} \mathrm{C}$ for $1 \mathrm{~h}$ then cooled to $0{ }^{\circ} \mathrm{C} .6 \mathrm{M} \mathrm{HCl}(5 \mathrm{~mL})$ was added and the resultant mixture was allowed to warm to $\mathrm{rt}$ over $18 \mathrm{~h}$ then the volatiles were removed in vacuo and the aqueous layer subjected to ion exchange chromatography (DOWEX 50WX8-200, eluent 1.0 M aq $\mathrm{NH}_{4} \mathrm{OH}$ ). Purification via flash column chromatography (eluent $\mathrm{CH}_{2} \mathrm{Cl}_{2} / \mathrm{MeOH} / 35 \%$ aq $\mathrm{NH}_{4} \mathrm{OH}, 25: 25: 1$ ) gave D-fagomine 1 as a white solid (30 mg, 65\%, >95:5 dr); mp 170-173 ${ }^{\circ} \mathrm{C}$; $\left\{\right.$ lit. $^{5} \mathrm{mp} 184-185^{\circ} \mathrm{C}$, lit. $\left.{ }^{1} \mathrm{mp} 186-188{ }^{\circ} \mathrm{C}\right\} ;[\alpha]_{\mathrm{D}}^{25}+14.9\left(c 1.0\right.$ in $\left.\mathrm{H}_{2} \mathrm{O}\right) ;\left\{\right.$ lit. $^{2,3}[\alpha]_{\mathrm{D}}+19.5(c$ 1.0 in $\left.\mathrm{H}_{2} \mathrm{O}\right)$; lit. $^{5}[\alpha]_{\mathrm{D}}^{20}+24.7\left(c 0.4\right.$ in $\left.\mathrm{H}_{2} \mathrm{O}\right)$; lit. $^{21,22}[\alpha]_{\mathrm{D}}^{20}+21.6\left(c 0.36\right.$ in $\left.\mathrm{H}_{2} \mathrm{O}\right)$; lit. $^{23}[\alpha]_{\mathrm{D}}^{20}+17.9(c 0.78$ in $\left.\left.\mathrm{H}_{2} \mathrm{O}\right)\right\} ; \delta_{\mathrm{H}}\left(400 \mathrm{MHz}, \mathrm{D}_{2} \mathrm{O}\right)^{33} 1.40-1.52\left(1 \mathrm{H}, \mathrm{m}, \mathrm{C}(2) H_{\mathrm{A}}\right), 1.97-2.04\left(1 \mathrm{H}, \mathrm{m}, \mathrm{C}(2) H_{\mathrm{B}}\right), 2.56(1 \mathrm{H}, \mathrm{ddd}$, $J$ 9.7, 6.7, 3.0, C(5)H), $2.63\left(1 \mathrm{H}, \mathrm{td}, J 12.8,2.6, \mathrm{C}(1) H_{\mathrm{A}}\right), 3.00-3.07\left(1 \mathrm{H}, \mathrm{m}, \mathrm{C}(1) H_{\mathrm{B}}\right), 3.19(1 \mathrm{H}, \mathrm{t}, J 9.7$, $\mathrm{C}(4) H), 3.55(1 \mathrm{H}, \mathrm{ddd}, J 11.5,9.0,5.0, \mathrm{C}(3) H), 3.65\left(1 \mathrm{H}, \mathrm{dd}, J 11.6,6.7, \mathrm{C}(6) H_{\mathrm{A}}\right), 3.86(1 \mathrm{H}, \mathrm{dd}, J 11.6,3.0$, $\left.\mathrm{C}(6) H_{\mathrm{B}}\right) ; \delta_{\mathrm{C}}\left(100 \mathrm{MHz}, \mathrm{D}_{2} \mathrm{O}\right)^{33} 35.5(C(2)), 45.4(C(1)), 63.7(C(5)), 64.5(C(6)), 76.0(C(4)), 76.1(C(3))$; $m / z\left(\mathrm{ESI}^{+}\right) 148\left([\mathrm{M}+\mathrm{H}]^{+}, 100 \%\right) ; \mathrm{HRMS}\left(\mathrm{ESI}^{+}\right) \mathrm{C}_{6} \mathrm{H}_{14} \mathrm{NO}_{3}{ }^{+}\left([\mathrm{M}+\mathrm{H}]^{+}\right)$requires 148.0968; found 148.0968. A sample of 1 was dissolved in $\mathrm{CHCl}_{3} / \mathrm{PrOH}(3: 1)$ and $2.0 \mathrm{M}$ aq $\mathrm{NaOH}$, and the aqueous layer was extracted with $\mathrm{CHCl}_{3} / \mathrm{PrOH}$ (3:1), then the combined organic extracts were concentrated in vacuo to give $\mathbf{1}$ as a white solid. The ${ }^{1} \mathrm{H}$ and ${ }^{13} \mathrm{C}$ NMR spectroscopic data for this sample were identical to those reported above.

\subsection{1. (4S,5R,6R)-4,5-Dihydroxy-6-(triisopropylsilyloxymethyl)piperidin-2-one 18}

Step 1: $\mathrm{Pd}(\mathrm{OH})_{2} / \mathrm{C}(20 \mathrm{mg}, 20 \mathrm{~mol} \%)$ was added to a vigorously stirred solution of 12 (100 mg, $0.154 \mathrm{mmol}$, $>95: 5 \mathrm{dr})$ in degassed $\mathrm{MeOH}(2 \mathrm{~mL})$. The reaction mixture was stirred under $\mathrm{H}_{2}(1 \mathrm{~atm})$ for $24 \mathrm{~h}$ then filtered through Celite (eluent $\mathrm{MeOH}$ ) and concentrated in vacuo. 
Step 2: $\mathrm{Na}(35 \mathrm{mg}, 1.5 \mathrm{mmol})$ was added to a stirred solution of naphthalene (237 mg, $1.85 \mathrm{mmol})$ in DME $(2 \mathrm{~mL})$ at $\mathrm{rt}$ and stirred at $\mathrm{rt}$ for $2 \mathrm{~h}$ then cooled to $-78{ }^{\circ} \mathrm{C}$. The residue from the previous step was dissolved in DME (4 mL) and added at $-78{ }^{\circ} \mathrm{C}$ and the resultant mixture was allowed to warm to rt over $18 \mathrm{~h}$. Satd aq $\mathrm{NH}_{4} \mathrm{Cl}(5 \mathrm{~mL})$ was added and the aqueous layer was extracted with $\mathrm{Et}_{2} \mathrm{O}(3 \times 5 \mathrm{~mL})$. The combined organic layers were washed with brine $(10 \mathrm{~mL})$ then dried and concentrated in vacuo. Purification via flash column chromatography (eluent $\mathrm{CHCl}_{3} / \mathrm{PrOH} / 35 \%$ aq $\mathrm{NH}_{4} \mathrm{OH}, 86: 14: 1$ ) gave $\mathbf{1 8}$ as a pale orange solid (37 mg, $76 \%,>95: 5 \mathrm{dr}) ; \operatorname{mp} 50-53{ }^{\circ} \mathrm{C} ;[\alpha]_{\mathrm{D}}^{25}+31.7\left(\right.$ c 1.0 in $\left.\mathrm{CHCl}_{3}\right)$; $v_{\max }(\mathrm{ATR}) 3399(\mathrm{O}-\mathrm{H}, \mathrm{N}-\mathrm{H}), 1651(\mathrm{C}=\mathrm{O}) ; \delta_{\mathrm{H}}$ $\left(500 \mathrm{MHz}, \mathrm{CDCl}_{3}, 18.9 \mathrm{mM}\right)^{31}$ 1.05-1.11 (21H, m, Si(CHMe $\left.)_{3}\right), 2.57\left(1 \mathrm{H}, \mathrm{dd}, J\right.$ 18.1, 4.1, C(3)H $\left.H_{\mathrm{A}}\right), 2.63$ $\left(1 \mathrm{H}, \mathrm{dd}, J 18.1,3.2, \mathrm{C}(3) H_{\mathrm{B}}\right), 3.02(1 \mathrm{H}$, br s, $\mathrm{OH}), 3.23(1 \mathrm{H}$, br s, $\mathrm{OH}), 3.63-3.75(3 \mathrm{H}, \mathrm{m}, \mathrm{C}(5) H, \mathrm{C}(6) H$, $\left.\mathrm{C}\left(1^{\prime}\right) H_{\mathrm{A}}\right), 3.97\left(1 \mathrm{H}, \mathrm{dd}, J 9.6,5.2, \mathrm{C}\left(1^{\prime}\right) H_{\mathrm{B}}\right), 4.18(1 \mathrm{H}, \mathrm{dd}, J 5.9,3.5, \mathrm{C}(4) H), 5.98(1 \mathrm{H}$, br s, $\mathrm{N} H) ; \delta_{\mathrm{C}}(125$ $\left.\mathrm{MHz}, \mathrm{CDCl}_{3}, 18.9 \mathrm{mM}\right)^{31} 11.7\left(\mathrm{Si}\left(\mathrm{CHMe}_{2}\right)_{3}\right), 17.9\left(\mathrm{Si}\left(\mathrm{CHM} e_{2}\right)_{3}\right), 36.9(C(3)), 54.3(C(6)), 66.0\left(C\left(1^{\prime}\right)\right), 67.4$ $(C(4)), 69.7(C(5)), 169.5(C(2)) ; \delta_{\mathrm{H}}\left(500 \mathrm{MHz}, \mathrm{CDCl}_{3}, 139 \mathrm{mM}\right)^{31} 1.03-1.09\left(21 \mathrm{H}, \mathrm{m}, \mathrm{Si}(\mathrm{CHMe})_{3}\right), 2.53$ $\left(1 \mathrm{H}, \mathrm{dd}, J 17.9,3.9, \mathrm{C}(3) H_{\mathrm{A}}\right), 2.59\left(1 \mathrm{H}, \mathrm{dd}, J 17.9,3.5, \mathrm{C}(3) H_{\mathrm{B}}\right), 3.58-3.72\left(3 \mathrm{H}, \mathrm{m}, \mathrm{C}(5) H, \mathrm{C}(6) H, \mathrm{C}\left(1^{\prime}\right) H_{\mathrm{A}}\right)$, $3.83\left(1 \mathrm{H}\right.$, br s, OH), $3.97\left(1 \mathrm{H}, \mathrm{dd}, J 9.6,4.4, \mathrm{C}\left(1^{\prime}\right) H_{\mathrm{B}}\right), 4.05(1 \mathrm{H}$, br s, OH), 4.11-4.16 (1H, m, C(4)H), 6.29 $(1 \mathrm{H}$, brs, $\mathrm{NH}) ; \delta_{\mathrm{C}}\left(125 \mathrm{MHz}, \mathrm{CDCl}_{3}, 18.9 \mathrm{mM}\right)^{31} 11.7\left(\mathrm{Si}\left(\mathrm{CHMe}_{2}\right)_{3}\right), 17.9\left(\mathrm{Si}(\mathrm{CHMe})_{3}\right), 37.0(C(3)), 54.9$ $(C(6)), 65.4\left(C\left(1^{\prime}\right)\right), 67.1(C(4)), 69.0(C(5)), 170.4(C(2))$; $\delta_{\mathrm{H}}\left(400 \mathrm{MHz}\right.$, acetone- $\left.d_{6}\right)$ 1.06-1.11 (21H, m, $\left.\mathrm{Si}(\mathrm{CHMe})_{3}\right), 2.35\left(1 \mathrm{H}, \mathrm{dd}, J\right.$ 17.2, 4.5, C(3)H $\left.H_{\mathrm{A}}\right), 2.45\left(1 \mathrm{H}, \mathrm{dd}, J 17.2,4.1, \mathrm{C}(3) H_{\mathrm{B}}\right), 3.56-3.62(1 \mathrm{H}, \mathrm{m}$, $\mathrm{C}(6) H), 3.80\left(1 \mathrm{H}\right.$, obsc dd, $J$ 9.9, 6.0, C(1') $\left.H_{\mathrm{A}}\right), 3.81-3.84(1 \mathrm{H}, \mathrm{m}, \mathrm{C}(5) H), 4.00(1 \mathrm{H}, \mathrm{dd}, J 9.9,4.2$, $\left.\mathrm{C}\left(1^{\prime}\right) H_{\mathrm{B}}\right), 4.09-4.14(1 \mathrm{H}, \mathrm{m}, \mathrm{C}(4) H), 4.19(1 \mathrm{H}, \mathrm{d}, J 5.9, \mathrm{OH}), 4.26(1 \mathrm{H}, \mathrm{d}, J 3.6, \mathrm{OH}), 6.32(1 \mathrm{H}, \mathrm{br} \mathrm{s}, \mathrm{N} H)$; $\delta_{\mathrm{C}}\left(125 \mathrm{MHz}\right.$, acetone- $\left.d_{6}\right) 12.6\left(\mathrm{Si}\left(C \mathrm{HMe}_{2}\right)_{3}\right), 18.3\left(\mathrm{Si}(\mathrm{CHMe})_{2}\right), 38.1(C(3)), 56.6(C(6)), 65.8\left(C\left(1^{\prime}\right)\right), 68.1$ $(C(4)), 68.8(C(5)), 169.9(C(2)) ; \mathrm{m} / z\left(\mathrm{ESI}^{+}\right) 318\left([\mathrm{M}+\mathrm{H}]^{+}, 100 \%\right), 340\left([\mathrm{M}+\mathrm{Na}]^{+}, 25 \%\right) ; \mathrm{HRMS}\left(\mathrm{ESI}^{+}\right)$ $\mathrm{C}_{15} \mathrm{H}_{32} \mathrm{NO}_{4} \mathrm{Si}^{+}\left([\mathrm{M}+\mathrm{H}]^{+}\right)$requires 318.2095; found 318.2096.

\subsection{1,2,5-Trideoxy-1,5-imino-D-ribo-hexitol [D-3-epi-fagomine] 2}

$\mathrm{BH}_{3} \cdot \mathrm{SMe}_{2}(2.0 \mathrm{M}$ in THF, $1.66 \mathrm{~mL}, 3.31 \mathrm{mmol})$ was added to a solution of $\mathbf{1 8}(150 \mathrm{mg}, 0.472 \mathrm{mmol},>95: 5$ dr) in THF $(6 \mathrm{~mL})$ at $0{ }^{\circ} \mathrm{C}$ and stirred at $0{ }^{\circ} \mathrm{C}$ for 30 min then warmed to rt over $2 \mathrm{~h}$ then $3 \mathrm{M}$ aq $\mathrm{NaOH}(2$ $\mathrm{mL})$ and $30 \%$ aq $\mathrm{H}_{2} \mathrm{O}_{2}(2 \mathrm{~mL})$ were added and the resultant mixture was heated at $85{ }^{\circ} \mathrm{C}$ for $1 \mathrm{~h}$ then cooled to $0{ }^{\circ} \mathrm{C} .6 \mathrm{M} \mathrm{HCl}(5 \mathrm{~mL})$ was added and the resultant mixture was allowed to warm to rt over $18 \mathrm{~h}$ then the volatiles were removed in vacuo and the aqueous layer subjected to ion exchange chromatography (DOWEX 50WX8-200, eluent 1.0 M aq $\mathrm{NH}_{4} \mathrm{OH}$ ). Purification via flash column chromatography (eluent $\mathrm{CH}_{2} \mathrm{Cl}_{2} / \mathrm{MeOH} / 35 \%$ aq $\left.\mathrm{NH}_{4} \mathrm{OH}, 25: 25: 1\right)$ gave D-3-epi-fagomine 2 as a white solid (36 mg, 52\%, >95:5 dr); 
mp 188-191 ${ }^{\circ} \mathrm{C} ;\left\{\right.$ lit. $\left.^{8 \mathrm{~d}} \mathrm{mp} 220-222{ }^{\circ} \mathrm{C}\right\} ;[\alpha]_{\mathrm{D}}^{25}+62.8\left(\right.$ c 1.0 in $\left.\mathrm{H}_{2} \mathrm{O}\right) ;\left\{\right.$ lit. $^{3,4,6}[\alpha]_{\mathrm{D}}+69.0\left(\right.$ c 0.5 in $\left.\mathrm{H}_{2} \mathrm{O}\right) ;$ lit. $^{8 \mathrm{~d}}$ $[\alpha]_{\mathrm{D}}^{26}+74.4\left(c 0.95\right.$ in $\left.\left.\mathrm{H}_{2} \mathrm{O}\right)\right\} ; \delta_{\mathrm{H}}\left(400 \mathrm{MHz}, \mathrm{D}_{2} \mathrm{O}\right)^{33} 1.67-1.77\left(1 \mathrm{H}, \mathrm{m}, \mathrm{C}(2) H_{\mathrm{A}}\right), 1.80-1.88\left(1 \mathrm{H}, \mathrm{m}, \mathrm{C}(2) H_{\mathrm{B}}\right)$, 2.73-2.91 (3H, m, C(1)H $\left.H_{2}, \mathrm{C}(5) H\right), 3.47(1 \mathrm{H}, \mathrm{dd}, J 10.1,3.0, \mathrm{C}(4) H), 3.62\left(1 \mathrm{H}, \mathrm{dd}, J 11.5,6.6, \mathrm{C}(6) H_{\mathrm{A}}\right)$, $3.82\left(1 \mathrm{H}, \mathrm{dd}, J 11.5,3.2, \mathrm{C}(6) H_{\mathrm{B}}\right), 4.09(1 \mathrm{H}$, app q, $J 3.1, \mathrm{C}(3) H) ; \delta_{\mathrm{C}}\left(100 \mathrm{MHz}, \mathrm{D}_{2} \mathrm{O}\right)^{33} 33.8(C(2)), 41.2$ $(C(1)), 58.6(C(5)), 64.9(C(6)), 70.7(C(3)), 72.4(C(4)) ; m / z\left(\mathrm{ESI}^{+}\right) 148\left([\mathrm{M}+\mathrm{H}]^{+}, 100 \%\right) ; \mathrm{HRMS}\left(\mathrm{ESI}^{+}\right)$ $\mathrm{C}_{6} \mathrm{H}_{14} \mathrm{NO}_{3}{ }^{+}\left([\mathrm{M}+\mathrm{H}]^{+}\right)$requires 148.0968; found 148.0968. A sample of 2 was dissolved in $\mathrm{CHCl}_{3} / \mathrm{PrOH}$ (3:1) and 2.0 $\mathrm{M}$ aq $\mathrm{NaOH}$, and the aqueous layer was extracted with $\mathrm{CHCl}_{3} / \mathrm{PrOH}$ (3:1), then the combined organic extracts were concentrated in vacuo to give 2 as a white solid. The ${ }^{1} \mathrm{H}$ and ${ }^{13} \mathrm{C}$ NMR spectroscopic data for this sample were identical to those reported above.

\subsection{3. tert-Butyl $(2 S, 3 R, \alpha R)$-2-hydroxy-3-[N-benzyl- $N$-( $\alpha$-methylbenzyl)amino]-4-}

\section{(triisopropylsilyloxy)butanoate 20}

Step 1: DMSO $(2.62 \mathrm{~mL}, 36.9 \mathrm{mmol})$ was added to a stirred solution of $(\mathrm{COCl})_{2}(1.56 \mathrm{~mL}, 18.5 \mathrm{mmol})$ in $\mathrm{CH}_{2} \mathrm{Cl}_{2}(23 \mathrm{~mL})$ at $-78{ }^{\circ} \mathrm{C}$ and the resultant mixture was allowed to stir at $-78{ }^{\circ} \mathrm{C}$ for $10 \mathrm{~min}$ then $7(5.00 \mathrm{~g}$, $9.23 \mathrm{mmol},>95: 5 \mathrm{dr})$ in $\mathrm{CH}_{2} \mathrm{Cl}_{2}(23 \mathrm{~mL})$ was added at $-78{ }^{\circ} \mathrm{C}$. The resultant mixture was allowed to stir at $-78{ }^{\circ} \mathrm{C}$ for $1 \mathrm{~h}$ then $\mathrm{Et}_{3} \mathrm{~N}(7.72 \mathrm{~mL}, 55.4 \mathrm{mmol})$ was added and the resultant mixture was allowed to stir at $-78{ }^{\circ} \mathrm{C}$ for $20 \mathrm{~min}$ then at $\mathrm{rt}$ for $20 \mathrm{~min}$. The organic layer was washed with $\mathrm{H}_{2} \mathrm{O}(2 \times 20 \mathrm{~mL})$ then the combined aqueous layers were extracted with $\mathrm{CH}_{2} \mathrm{Cl}_{2}(2 \times 20 \mathrm{~mL})$ then the combined organic layers were dried and concentrated in vacuo to give 19 (>95\% conversion) as a yellow viscous oil.

Step 2: $\mathrm{NaBH}_{4}(349 \mathrm{mg}, 9.23 \mathrm{mmol})$ was added to a stirred solution of the residue of 19 in $\mathrm{MeOH}(307 \mathrm{~mL})$ at $-20{ }^{\circ} \mathrm{C}$ and the resultant mixture was stirred at $-20{ }^{\circ} \mathrm{C}$ for $2 \mathrm{~h}$ then concentrated in vacuo to give a 93:7 mixture of 20 and 7, respectively. Purification via flash column chromatography (eluent $30-40{ }^{\circ} \mathrm{C}$ petroleum ether/Et $\left.{ }_{2} \mathrm{O}, 25: 1\right)$ gave a 88:12 mixture of $\mathbf{2 0}$ and 7, respectively, as pale yellow viscous oil (1.58 g, 32\%, 88:12 dr). Further elution gave 20 as a pale yellow viscous oil $(2.76 \mathrm{~g}, 55 \%,>95: 5 \mathrm{dr})$; $[\alpha]_{\mathrm{D}}^{25}-26.5(c 1.0$ in $\left.\mathrm{CHCl}_{3}\right) ; v_{\max }(\mathrm{ATR}) 3510(\mathrm{O}-\mathrm{H}), 1723(\mathrm{C}=\mathrm{O}) ; \delta_{\mathrm{H}}\left(400 \mathrm{MHz}, \mathrm{CDCl}_{3}\right)$ 1.06-1.14 (21H, m, Si(CHMe2) $\left.)\right)$, $1.41\left(9 \mathrm{H}, \mathrm{s}, \mathrm{CM} e_{3}\right), 1.46(3 \mathrm{H}, \mathrm{d}, J$ 7.0, C( $\left.\alpha) M e\right), 3.29(1 \mathrm{H}, \mathrm{q}, J$ 6.4, C(3)H), $3.74(1 \mathrm{H}, \mathrm{d}, J 3.4, \mathrm{OH})$, 3.86-3.99 (4H, m, C(2)H, C(4)H $\left.H_{2}, \mathrm{NCH}_{\mathrm{A}} \mathrm{H}_{\mathrm{B}} \mathrm{Ph}\right), 4.04\left(1 \mathrm{H}, \mathrm{d}, J 13.9, \mathrm{NCH}_{\mathrm{A}} H_{\mathrm{B}} \mathrm{Ph}\right), 4.18(1 \mathrm{H}, \mathrm{q}, J$ 7.0, $\mathrm{C}(\alpha) H), \quad 7.18-7.32(10 \mathrm{H}, \quad \mathrm{m}, \quad P h) ; \quad \delta_{\mathrm{C}}\left(100 \mathrm{MHz}, \mathrm{CDCl}_{3}\right) 11.9\left(\mathrm{Si}\left(\mathrm{CHMe}_{2}\right)_{3}\right), 17.4(\mathrm{C}(\alpha) M e), 18.1$ $\left(\mathrm{Si}(\mathrm{CHMe})_{3}\right), 27.9\left(\mathrm{CMe}_{3}\right), 51.0\left(\mathrm{NCH}_{2} \mathrm{Ph}\right), 58.8(C(\alpha)), 61.5(C(3)), 61.7(C(4)), 70.1(C(2)), 81.5\left(C \mathrm{Me}_{3}\right)$, 126.8, 126.9, 127.9, 128.2, $128.7(o, m, p-P h), 140.7,143.7(i-P h), 172.9(C(1)) ; m / z\left(\mathrm{ESI}^{+}\right) 542\left([\mathrm{M}+\mathrm{H}]^{+}\right.$, $100 \%) ; \mathrm{HRMS}\left(\mathrm{ESI}^{+}\right) \mathrm{C}_{32} \mathrm{H}_{52} \mathrm{NO}_{4} \mathrm{Si}^{+}\left([\mathrm{M}+\mathrm{H}]^{+}\right)$requires 542.3660; found 542.3650. 


\subsection{4. $(2 S, 3 R, \alpha R)-2-B e n z y l o x y-3-[N$-benzyl- $N$-( $\alpha$-methylbenzyl)amino]-4-(triisopropylsilyloxy)butan-1-}

ol 21

Method A - Step 1: A solution of $20(2.76 \mathrm{~g}, 5.09 \mathrm{mmol},>95: 5 \mathrm{dr})$ in THF (45 mL) was added to a stirred suspension of $\mathrm{NaH}(60 \%$ in mineral oil, $408 \mathrm{mg}, 10.2 \mathrm{mmol})$ in $\mathrm{THF}(45 \mathrm{~mL})$ at $0{ }^{\circ} \mathrm{C}$ and the resultant mixture was stirred at $0{ }^{\circ} \mathrm{C}$ for $1 \mathrm{~h}$. $\mathrm{BnBr}(1.21 \mathrm{~mL}, 10.2 \mathrm{mmol})$ was added and the resultant mixture was allowed to warm to rt over $18 \mathrm{~h}$ then satd aq $\mathrm{NH}_{4} \mathrm{Cl}(10 \mathrm{~mL})$ and $\mathrm{Et}_{2} \mathrm{O}(50 \mathrm{~mL})$ were added. The resultant mixture was washed with brine $(300 \mathrm{~mL})$ and the aqueous layers were extracted with $\mathrm{Et}_{2} \mathrm{O}(3 \times 50 \mathrm{~mL})$ then the combined organic layers were dried and concentrated in vacuo.

Method A - Step 2: DIBAL-H (1.0 M in $\left.\mathrm{CH}_{2} \mathrm{Cl}_{2}, 20.4 \mathrm{~mL}, 20.4 \mathrm{mmol}\right)$ was added to a stirred solution of the residue from the previous step in $\mathrm{CH}_{2} \mathrm{Cl}_{2}(80 \mathrm{~mL})$ at $-78{ }^{\circ} \mathrm{C}$ then stirred at $\mathrm{rt}$ for $2 \mathrm{~h}$. Satd aq $\mathrm{NH}_{4} \mathrm{Cl}(10 \mathrm{~mL})$ was added and the resultant mixture was filtered through $C$ elite ${ }^{\circledR}\left(\right.$ eluent $\mathrm{CH}_{2} \mathrm{Cl}_{2}$ ) then dried and concentrated in vacuo. Purification via flash column chromatography (eluent $30-40{ }^{\circ} \mathrm{C}$ petroleum ether/EtOAc, 12:1) gave 21 as a pale yellow viscous oil (2.73 g, 95\% from 20, >95:5 dr); $\alpha]_{\mathrm{D}}^{25}-9.1$ (c 1.0 in $\left.\mathrm{CHCl}_{3}\right) ; v_{\max }(\mathrm{ATR}) 3591(\mathrm{O}-\mathrm{H}) ; \delta_{\mathrm{H}}\left(400 \mathrm{MHz}, \mathrm{CDCl}_{3}\right)$ 1.04-1.18 (21H, m, Si $\left.\left(\mathrm{CHMe}_{2}\right)_{3}\right), 1.44(3 \mathrm{H}, \mathrm{d}$, $J$ 6.9, C( $(\alpha) M e), 2.39(1 \mathrm{H}$, br s, $\mathrm{OH}), 2.95-3.01(1 \mathrm{H}, \mathrm{m}, \mathrm{C}(3) H), 3.20-3.29\left(1 \mathrm{H}, \mathrm{m}, \mathrm{C}(1) H_{\mathrm{A}}\right), 3.56-3.63(2 \mathrm{H}$, m, C(1) $\left.H_{\mathrm{B}}, \mathrm{C}(2) H\right), 3.72\left(1 \mathrm{H}, \mathrm{d}, J 13.2, \mathrm{NCH}_{\mathrm{A}} \mathrm{H}_{\mathrm{B}} \mathrm{Ph}\right), 4.05\left(1 \mathrm{H}, \mathrm{dd}, J\right.$ 9.5, 5.1, C(4)H $\left.H_{\mathrm{A}}\right), 4.09-4.16(1 \mathrm{H}, \mathrm{m}$, $\left.\mathrm{C}(4) H_{\mathrm{B}}\right), 4.19(1 \mathrm{H}, \mathrm{q}, J 6.9, \mathrm{C}(\alpha) H), 4.35\left(1 \mathrm{H}, \mathrm{d}, J 13.2, \mathrm{NCH}_{\mathrm{A}} H_{\mathrm{B}} \mathrm{Ph}\right), 4.43\left(1 \mathrm{H}, \mathrm{d}, J 11.7, \mathrm{OCH}_{\mathrm{A}} \mathrm{H}_{\mathrm{B}} \mathrm{Ph}\right)$, $4.61\left(1 \mathrm{H}, \mathrm{d}, J\right.$ 11.7, $\left.\mathrm{OCH}_{\mathrm{A}} H_{\mathrm{B}} \mathrm{Ph}\right), 7.21-7.39(15 \mathrm{H}, \mathrm{m}, \mathrm{Ph}) ; \delta_{\mathrm{C}}\left(100 \mathrm{MHz}, \mathrm{CDCl}_{3}\right) 12.0\left(\mathrm{Si}\left(\mathrm{CHMe}_{2}\right)_{3}\right), 13.7$ $(\mathrm{C}(\alpha) M e), 18.1\left(\mathrm{Si}(\mathrm{CHMe})_{3}\right), 52.0\left(\mathrm{NCH}_{2} \mathrm{Ph}\right), 56.4(C(\alpha)), 59.3(C(3)), 61.3(C(4)), 61.9(C(1)), 72.3$ $\left(\mathrm{OCH}_{2} \mathrm{Ph}\right), 79.6(C(2)), 126.9,127.2,127.3,128.1,128.2$, 128.3, $129.4(o, m, p-P h), 139.0,141.0,143.9$, $(i-P h) ; m / z\left(\mathrm{ESI}^{+}\right) 562\left([\mathrm{M}+\mathrm{H}]^{+}, 100 \%\right) ; \operatorname{HRMS}\left(\mathrm{ESI}^{+}\right) \mathrm{C}_{35} \mathrm{H}_{52} \mathrm{NO}_{3} \mathrm{Si}^{+}\left([\mathrm{M}+\mathrm{H}]^{+}\right)$requires 562.3711; found 562.3709 .

Method B - Step 1: DMSO (14.1 mL, $199 \mathrm{mmol})$ was added to a stirred solution of $(\mathrm{COCl})_{2}(8.42 \mathrm{~mL}, 99.5$ $\mathrm{mmol})$ in $\mathrm{CH}_{2} \mathrm{Cl}_{2}(125 \mathrm{~mL})$ at $-78^{\circ} \mathrm{C}$ and the resultant mixture was allowed to stir at $-78{ }^{\circ} \mathrm{C}$ for 10 min then $7(27.0 \mathrm{~g}, 49.8 \mathrm{mmol},>95: 5 \mathrm{dr})$ in $\mathrm{CH}_{2} \mathrm{Cl}_{2}(125 \mathrm{~mL})$ was added at $-78{ }^{\circ} \mathrm{C}$. The resultant mixture was allowed to stir at $-78{ }^{\circ} \mathrm{C}$ for $1 \mathrm{~h}$ then $\mathrm{Et}_{3} \mathrm{~N}(41.7 \mathrm{~mL}, 299 \mathrm{mmol})$ was added and the resultant mixture was allowed to stir at $-78{ }^{\circ} \mathrm{C}$ for $20 \mathrm{~min}$ then at $\mathrm{rt}$ for $20 \mathrm{~min}$. The organic layer was washed with $\mathrm{H}_{2} \mathrm{O}(2 \times 150$ $\mathrm{mL})$ then the combined aqueous layers were extracted with $\mathrm{CH}_{2} \mathrm{Cl}_{2}(2 \times 200 \mathrm{~mL})$ then the combined organic layers were dried and concentrated in vacuo to give 19 (>95\% conversion) as a yellow viscous oil. 
Method B - Step 2: $\mathrm{NaBH}_{4}(1.88 \mathrm{~g}, 49.8 \mathrm{mmol})$ was added to a stirred solution of the residue of 19 in $\mathrm{MeOH}$ $(1.60 \mathrm{~L})$ at $-20{ }^{\circ} \mathrm{C}$ and the resultant mixture was stirred at $-20{ }^{\circ} \mathrm{C}$ for $2 \mathrm{~h}$ then concentrated in vacuo to give 20.

Method B - Step 3: A solution of the residue of $\mathbf{2 0}$ from the previous in THF (300 mL) was added to a stirred suspension of $\mathrm{NaH}(60 \%$ in mineral oil, $3.98 \mathrm{~g}, 99.6 \mathrm{mmol})$ in $\mathrm{THF}(300 \mathrm{~mL})$ at $0{ }^{\circ} \mathrm{C}$ and the resultant mixture was stirred at $0{ }^{\circ} \mathrm{C}$ for $1 \mathrm{~h}$. $\mathrm{BnBr}(11.9 \mathrm{~mL}, 99.6 \mathrm{mmol})$ was added and the resultant mixture was allowed to warm to rt over $18 \mathrm{~h}$ then satd aq $\mathrm{NH}_{4} \mathrm{Cl}(20 \mathrm{~mL})$ and $\mathrm{Et}_{2} \mathrm{O}(100 \mathrm{~mL})$ were added. The resultant mixture was washed with brine $(100 \mathrm{~mL})$ and the aqueous layers were extracted with $\mathrm{Et}_{2} \mathrm{O}(3 \times 150 \mathrm{~mL})$ then the combined organic layers were dried and concentrated in vacuo.

Method B - Step 4: DIBAL-H (1.0 M in $\left.\mathrm{CH}_{2} \mathrm{Cl}_{2}, 200 \mathrm{~mL}, 200 \mathrm{mmol}\right)$ was added to a stirred solution of the residue from the previous step in $\mathrm{CH}_{2} \mathrm{Cl}_{2}(600 \mathrm{~mL})$ at $-78{ }^{\circ} \mathrm{C}$ then stirred at $\mathrm{rt}$ for $2 \mathrm{~h}$. Satd aq $\mathrm{NH}_{4} \mathrm{Cl}(50$ $\mathrm{mL}$ ) was added and the resultant mixture was filtered through Celite ${ }^{\circledR}$ (eluent $\mathrm{CH}_{2} \mathrm{Cl}_{2}$ ) then dried and concentrated in vacuo. Purification via flash column chromatography (eluent 30-40 ${ }^{\circ} \mathrm{C}$ petroleum ether/EtOAc, 12:1) gave 21 as a pale yellow viscous oil (23.9 g, 85\% from 7, >95:5 dr).

\subsection{Ethyl $(3 R, 4 S, 5 R, \alpha R)-3-h y d r o x y-4-b e n z y l o x y-5-[N$-benzyl- $N$-( $\alpha$-methylbenzyl)amino]-6-}

\section{(triisoproylsilyloxy)hexanoate 22 and ethyl $(3 S, 4 S, 5 R, \alpha R)-3$-hydroxy-4-benzyloxy-5-[N-benzyl- $N$ - $(\alpha-$}

\section{methylbenzyl)amino]-6-(triisoproylsilyloxy)hexanoate 23}

Step 1: DMSO $(0.51 \mathrm{~mL}, 7.12 \mathrm{mmol})$ was added to a stirred solution of $(\mathrm{COCl})_{2}(0.30 \mathrm{~mL}, 3.56 \mathrm{mmol})$ in $\mathrm{CH}_{2} \mathrm{Cl}_{2}(10 \mathrm{~mL})$ at $-78{ }^{\circ} \mathrm{C}$ and the resultant mixture was allowed to stir at $-78{ }^{\circ} \mathrm{C}$ for $10 \mathrm{~min}$ then $21(1.00 \mathrm{~g}$, $1.78 \mathrm{mmol},>95: 5 \mathrm{dr})$ in $\mathrm{CH}_{2} \mathrm{Cl}_{2}(10 \mathrm{~mL})$ was added at $-78{ }^{\circ} \mathrm{C}$. The resultant mixture was allowed to stir at $-78{ }^{\circ} \mathrm{C}$ for $1 \mathrm{~h}$ then $\mathrm{Et}_{3} \mathrm{~N}(1.49 \mathrm{~mL}, 10.7 \mathrm{mmol})$ was added and the resultant mixture was allowed to stir at $-78{ }^{\circ} \mathrm{C}$ for $20 \mathrm{~min}$ then at $\mathrm{rt}$ for $20 \mathrm{~min}$. The organic layer was washed with $\mathrm{H}_{2} \mathrm{O}(2 \times 15 \mathrm{~mL})$ then the combined aqueous layers were extracted with $\mathrm{CH}_{2} \mathrm{Cl}_{2}(2 \times 15 \mathrm{~mL})$ then the combined organic layers were dried and concentrated in vacuo.

Step 2: $\mathrm{BuLi}(2.2 \mathrm{M}$ in hexanes, $1.13 \mathrm{~mL}, 2.49 \mathrm{mmol})$ was added to a stirred solution of ${ }^{\mathrm{i}} \mathrm{Pr}_{2} \mathrm{NH}(0.35 \mathrm{~mL}$, $2.49 \mathrm{mmol})$ in $\mathrm{THF}(5 \mathrm{~mL})$ at $-78{ }^{\circ} \mathrm{C}$ and the resultant mixture was stirred at $-78{ }^{\circ} \mathrm{C}$ for $20 \mathrm{~min}$ then EtOAc $(0.24 \mathrm{~mL}, 2.49 \mathrm{mmol})$ was added at $-78{ }^{\circ} \mathrm{C}$ and the resultant mixture was stirred at $-78{ }^{\circ} \mathrm{C}$ for $30 \mathrm{~min}$. A solution of the residue from the previous step in THF $(5 \mathrm{~mL})$ was added at $-78^{\circ} \mathrm{C}$ and the resultant mixture was allowed to stir at $-78{ }^{\circ} \mathrm{C}$ for $1 \mathrm{~h}$ then at $0{ }^{\circ} \mathrm{C}$ for $2 \mathrm{~h}$ before satd aq $\mathrm{NH}_{4} \mathrm{Cl}(2 \mathrm{~mL})$ and $\mathrm{Et}_{2} \mathrm{O}(10 \mathrm{~mL})$ was added. The aqueous layer was extracted with $\mathrm{Et}_{2} \mathrm{O}(3 \times 10 \mathrm{~mL})$ then the combined organic layers were 
washed with brine $(25 \mathrm{~mL})$ then were dried and concentrated in vacuo to give a 46:54 mixture of $\mathbf{2 2}$ and $\mathbf{2 3}$, respectively. Purification via flash column chromatography (eluent $30-40{ }^{\circ} \mathrm{C}$ petroleum ether/acetone, 10:1) gave a 46:54 mixture of $\mathbf{2 2}$ and $\mathbf{2 3}$, respectively, as a colourless viscous oil (902 $\mathrm{mg}, \mathbf{7 2 \%}$, 54:46 dr). Data for mixture: $v_{\max }(\mathrm{ATR}) 3587(\mathrm{O}-\mathrm{H}), 1733(\mathrm{C}=\mathrm{O}) ; \mathrm{m} / \mathrm{z}\left(\mathrm{ESI}^{+}\right) 648\left([\mathrm{M}+\mathrm{H}]^{+}, 100 \%\right)$; HRMS $\left(\mathrm{ESI}^{+}\right)$ $\mathrm{C}_{39} \mathrm{H}_{58} \mathrm{NO}_{5} \mathrm{Si}^{+}\left([\mathrm{M}+\mathrm{H}]^{+}\right)$requires 648.4079 ; found 648.4076. Data for 22: $\delta_{\mathrm{H}}\left(400 \mathrm{MHz}, \mathrm{CDCl}_{3}\right) 1.07-1.16$ $\left(21 \mathrm{H}, \mathrm{m}, \mathrm{Si}(\mathrm{CHMe})_{3}\right), 1.20-1.26\left(3 \mathrm{H}, \mathrm{m}, \mathrm{OCH}_{2} \mathrm{CH}_{3}\right), 1.40-1.46(3 \mathrm{H}, \mathrm{m}, \mathrm{C}(\alpha) M e), 1.87(1 \mathrm{H}, \mathrm{dd}, J$ 15.7, 8.8, $\left.\mathrm{C}(2) H_{\mathrm{A}}\right), 2.11\left(1 \mathrm{H}, \mathrm{dd}, J 15.7,4.4, \mathrm{C}(2) H_{\mathrm{B}}\right), 3.07-3.13(1 \mathrm{H}, \mathrm{m}, \mathrm{C}(5) H), 3.55(1 \mathrm{H}, \mathrm{dd}, J 5.9,2.5, \mathrm{C}(4) H)$, 3.61-3.68 (1H, m, $\left.\mathrm{NCH}_{\mathrm{A}} \mathrm{H}_{\mathrm{B}} \mathrm{Ph}\right), 4.00-4.30\left(5 \mathrm{H}, \mathrm{m}, \mathrm{C}(6) \mathrm{H}_{2}, \mathrm{C}(\alpha) H, \mathrm{OCH}_{2} \mathrm{CH}_{3}\right), 4.31-4.37(1 \mathrm{H}, \mathrm{m}, \mathrm{C}(3) H)$, 4.46-4.53 $\left(1 \mathrm{H}, \mathrm{m}, \mathrm{NCH}_{\mathrm{A}} H_{\mathrm{B}} \mathrm{Ph}\right), 4.57\left(1 \mathrm{H}, \mathrm{d}, J 11.7, \mathrm{OCH}_{\mathrm{A}} \mathrm{H}_{\mathrm{B}} \mathrm{Ph}\right), 4.69\left(1 \mathrm{H}, \mathrm{d}, J 11.7, \mathrm{OCH}_{\mathrm{A}} H_{\mathrm{B}} \mathrm{Ph}\right)$, 7.19-7.42 (15H, m, Ph); $\delta_{\mathrm{C}}\left(100 \mathrm{MHz}, \mathrm{CDCl}_{3}\right)$ [selected data] $12.0\left(\mathrm{Si}\left(\mathrm{CHMe}_{2}\right)_{3}\right), 14.2\left(\mathrm{OCH}_{2} \mathrm{Me}\right), 18.2$ $\left(\mathrm{Si}(\mathrm{CHMe})_{3}\right), 38.7(C(2)), 52.0\left(\mathrm{NCH}_{2} \mathrm{Ph}\right), 55.6(C(\alpha)), 57.8(C(5)), 60.9(C(6)), 68.2(C(3)), 73.6$ $\left(\mathrm{OCH}_{2} \mathrm{Ph}\right), 81.0(C(4))$. Data for $23: \delta_{\mathrm{H}}\left(400 \mathrm{MHz}, \mathrm{CDCl}_{3}\right)$ 1.07-1.16 (21H, m, Si $\left.\left(\mathrm{CHMe}_{2}\right)_{3}\right), 1.20-1.26(3 \mathrm{H}$, m, $\left.\mathrm{OCH}_{2} \mathrm{CH}_{3}\right), 1.40-1.46(3 \mathrm{H}, \mathrm{m}, \mathrm{C}(\alpha) M e), 1.99\left(1 \mathrm{H}, \mathrm{dd}, J 15.4,5.0, \mathrm{C}(2) H_{\mathrm{A}}\right), 2.24(1 \mathrm{H}, \mathrm{dd}, J 15.4,8.3$, $\left.\mathrm{C}(2) H_{\mathrm{B}}\right), 3.02(1 \mathrm{H}, \mathrm{td}, J 6.8,3.4, \mathrm{C}(5) H), 3.50(1 \mathrm{H}, \mathrm{t}, J 3.4, \mathrm{C}(4) H), 3.74\left(1 \mathrm{H}, \mathrm{d}, J 13.4, \mathrm{NCH}_{\mathrm{A}} \mathrm{H}_{\mathrm{B}} \mathrm{Ph}\right)$, 3.84-3.91 (1H, m, C(3)H), 4.00-4.30 (5H, m, C(6)H $\left., \mathrm{C}(\alpha) H, \mathrm{OCH}_{2} \mathrm{CH}_{3}\right), 4.41\left(1 \mathrm{H}, \mathrm{d}, J 13.4, \mathrm{NCH}_{\mathrm{A}} H_{\mathrm{B}} \mathrm{Ph}\right)$, 4.46-4.53 (1H, m, OCH $\left.H_{\mathrm{A}} \mathrm{H}_{\mathrm{B}} \mathrm{Ph}\right), 4.63\left(1 \mathrm{H}, \mathrm{d}, J\right.$ 11.6, $\left.\mathrm{OCH}_{\mathrm{A}} H_{\mathrm{B}} \mathrm{Ph}\right), 7.19-7.42(15 \mathrm{H}, \mathrm{m}, P h) ; \delta_{\mathrm{C}}(100 \mathrm{MHz}$, $\left.\mathrm{CDCl}_{3}\right)$ [selected data] $12.0\left(\mathrm{Si}\left(\mathrm{CHMe}_{2}\right)_{3}\right), 14.2\left(\mathrm{OCH}_{2} \mathrm{CH}_{3}\right), 18.2\left(\mathrm{Si}(\mathrm{CHMe})_{3}\right), 38.9(\mathrm{C}(2)), 51.9$ $\left(\mathrm{NCH}_{2} \mathrm{Ph}\right), 55.6(C(\alpha)), 60.0(C(5)), 60.9(C(6)), 69.8(C(3)), 75.2\left(\mathrm{OCH}_{2} \mathrm{Ph}\right), 81.0(C(4))$.

\subsection{6. (4R,5S,6R)-4,5-Dihydroxy-6-(triisopropylsilyloxymethyl)piperidin-2-one 24 and (4S,5S,6R)-4,5-dihydroxy-6-(triisopropylsilyloxymethyl)piperidin-2-one 25}

Step 1: $\mathrm{Pd}(\mathrm{OH}) 2 / \mathrm{C}(100 \mathrm{mg}, 20 \mathrm{~mol} \%)$ was added to a vigorously stirred solution of a 46:54 mixture of 22 and 23, respectively, (500 $\mathrm{mg}, 0.772 \mathrm{mmol}, 54: 46 \mathrm{dr}$ ) in degassed $\mathrm{MeOH}$ (4 mL). The reaction mixture was stirred under $\mathrm{H}_{2}$ (5 atm) for $24 \mathrm{~h}$ then filtered through Celite (eluent $\mathrm{MeOH}$ ) and concentrated in vacuo.

Step 2: $\mathrm{Na}(177 \mathrm{mg}, 7.72 \mathrm{mmol})$ was added to a stirred solution of naphthalene $(1.19 \mathrm{~g}, 9.26 \mathrm{mmol}) \mathrm{in} \mathrm{THF}$ $(10 \mathrm{~mL})$ at $\mathrm{rt}$ and stirred at $\mathrm{rt}$ for $2 \mathrm{~h}$, then cooled to $-78{ }^{\circ} \mathrm{C}$. The residue from the previous step was dissolved in THF $(10 \mathrm{~mL})$ and added at $-78{ }^{\circ} \mathrm{C}$ and the resultant mixture was allowed to warm to $0{ }^{\circ} \mathrm{C}$ over $3 \mathrm{~h}$. Satd aq $\mathrm{NH}_{4} \mathrm{Cl}(10 \mathrm{~mL})$ was added and the aqueous layer was extracted with $\mathrm{Et}_{2} \mathrm{O}(3 \times 20 \mathrm{~mL})$. The combined organic layers were washed with brine $(25 \mathrm{~mL})$ then dried and concentrated in vacuo. Purification via flash column chromatography (eluent $\mathrm{CHCl}_{3} /{ }^{j} \mathrm{PrOH} / 35 \%$ aq $\mathrm{NH}_{4} \mathrm{OH}, 89: 11: 1$ ) to give 24 as colourless viscous oil (76 mg, 31\%, >95:5 dr); $[\alpha]_{\mathrm{D}}^{25}+27.1$ ( c 1.0 in $\left.\mathrm{CHCl}_{3}\right) ; v_{\max }(\mathrm{ATR}) 3402(\mathrm{O}-\mathrm{H}), 1646(\mathrm{C}=\mathrm{O})$; $\delta_{\mathrm{H}}$ 
$\left(500 \mathrm{MHz}, \mathrm{CDCl}_{3}, 18.9 \mathrm{mM}\right)^{31} 1.04-1.18\left(21 \mathrm{H}, \mathrm{m}, \mathrm{Si}(\mathrm{CHMe})_{3}\right), 2.61\left(1 \mathrm{H}, \mathrm{dd}, J\right.$ 17.2, 10.2, C(3)H $\left.H_{\mathrm{A}}\right), 2.67$ $\left(1 \mathrm{H}, \mathrm{dd}, J 17.2,6.6, \mathrm{C}(3) H_{\mathrm{B}}\right), 2.79(1 \mathrm{H}$, br s, $\mathrm{OH}), 2.98(1 \mathrm{H}$, br s, $\mathrm{OH}), 3.50-3.55(1 \mathrm{H}, \mathrm{m}, \mathrm{C}(6) H), 3.91(1 \mathrm{H}$, dd, $\left.J 10.1,4.4, \mathrm{C}\left(1^{\prime}\right) H_{\mathrm{A}}\right), 3.91\left(1 \mathrm{H}, \mathrm{dd}, J 10.1,8.0, \mathrm{C}\left(1^{\prime}\right) H_{\mathrm{B}}\right), 4.04-4.12(2 \mathrm{H}, \mathrm{m}, \mathrm{C}(4) H, \mathrm{C}(5) H), 5.95(1 \mathrm{H}, \mathrm{br}$ s, $\mathrm{NH}) ; \delta_{\mathrm{C}}\left(125 \mathrm{MHz}, \mathrm{CDCl}_{3}, 18.9 \mathrm{mM}\right)^{31} 11.8\left(\mathrm{Si}\left(\mathrm{CHMe}_{2}\right)_{3}\right), 17.9\left(\mathrm{Si}(\mathrm{CHMe})_{3}\right), 35.5(C(3)), 55.2(C(6))$, $63.9\left(C\left(1^{\prime}\right)\right), 67.7(C(4)), 67.7(C(5)), 170.3(C(2)) ; \delta_{\mathrm{H}}\left(500 \mathrm{MHz}, \mathrm{CDCl}_{3}, 139 \mathrm{mM}\right)^{31} 1.01-1.14(21 \mathrm{H}, \mathrm{m}$, $\left.\mathrm{Si}\left(\mathrm{CHMe}_{2}\right)_{3}\right), 2.55-2.67\left(2 \mathrm{H}, \mathrm{m}, \mathrm{C}(3) \mathrm{H}_{2}\right), 3.47-3.52(1 \mathrm{H}, \mathrm{m}, \mathrm{C}(6) H), 3.79(1 \mathrm{H}, \mathrm{br} \mathrm{s}, \mathrm{OH}), 3.85-3.91(2 \mathrm{H}, \mathrm{m}$, $\left.\mathrm{C}\left(1^{\prime}\right) H_{2}\right), 4.00-4.07(2 \mathrm{H}, \mathrm{m}, \mathrm{C}(4) H, \mathrm{C}(5) H), 4.19(1 \mathrm{H}$, br s, OH),5.95 (1H, br s, $\mathrm{NH})$; $\delta_{\mathrm{C}}\left(125 \mathrm{MHz}, \mathrm{CDCl}_{3}\right.$, $18.9 \mathrm{mM})^{31} 11.8\left(\mathrm{Si}\left(\mathrm{CHMe}_{2}\right)_{3}\right), 17.9\left(\mathrm{Si}(\mathrm{CHMe})_{3}\right), 35.3(C(3)), 55.7(C(6)), 63.8\left(C\left(1^{\prime}\right)\right), 67.3(C(5)), 67.6$ $(C(4)), 171.2(C(2)) ; m / z\left(\mathrm{ESI}^{+}\right) 318\left([\mathrm{M}+\mathrm{H}]^{+}, 100 \%\right), 340\left([\mathrm{M}+\mathrm{Na}]^{+}, 74 \%\right) ; \mathrm{HRMS}\left(\mathrm{ESI}^{+}\right) \mathrm{C}_{15} \mathrm{H}_{32} \mathrm{NO}_{4} \mathrm{Si}^{+}$ $\left([\mathrm{M}+\mathrm{H}]^{+}\right)$requires 318.2095; found 318.2088. Further elution gave 25 as a pale orange solid (85 mg, 35\%, >95:5 dr); mp 140-141 ${ }^{\circ} \mathrm{C}$; $[\alpha]_{\mathrm{D}}^{25}+29.4$ (c 1.0 in $\left.\mathrm{CHCl}_{3}\right) ; v_{\max }(\mathrm{ATR}) 3398(\mathrm{O}-\mathrm{H}), 1644(\mathrm{C}=\mathrm{O}) ; \delta_{\mathrm{H}}(500$ $\left.\mathrm{MHz}, \mathrm{CDCl}_{3}, 18.9 \mathrm{mM}\right)^{31} 1.04-1.11\left(21 \mathrm{H}, \mathrm{m}, \mathrm{Si}(\mathrm{CHMe})_{3}\right), 2.39\left(1 \mathrm{H}, \mathrm{dd}, J\right.$ 17.9, 3.4, C(3)H $\left.H_{\mathrm{A}}\right), 2.80(1 \mathrm{H}$, brs, $\mathrm{OH}), 2.86\left(1 \mathrm{H}, \mathrm{dd}, J 17.9,4.6, \mathrm{C}(3) H_{\mathrm{B}}\right), 3.49(1 \mathrm{H}, \mathrm{br} \mathrm{s}, \mathrm{OH}), 3.78-3.82(1 \mathrm{H}, \mathrm{m}, \mathrm{C}(6) H), 3.92-3.99(2 \mathrm{H}$, m, C(1') H2), 3.99-4.03 (1H, m, C(5)H), 4.15-4.20 (1H, m, C(4)H), $5.93\left(1 \mathrm{H}\right.$, br s, NH); $\delta_{\mathrm{C}}(125 \mathrm{MHz}$, $\left.\mathrm{CDCl}_{3}, 18.9 \mathrm{mM}\right)^{31} 11.7\left(\mathrm{Si}\left(\mathrm{CHMe}_{2}\right)_{3}\right), 17.9\left(\mathrm{Si}(\mathrm{CHMe})_{3}\right), 36.1(C(3)), 52.9(C(6)), 64.5\left(C\left(1^{\prime}\right)\right), 67.1$ $(C(4)), 69.1(C(5)), 170.8(C(2)) ; \delta_{\mathrm{H}}\left(500 \mathrm{MHz}, \mathrm{CDCl}_{3}, 139 \mathrm{mM}\right)^{31} 1.00-1.19\left(21 \mathrm{H}, \mathrm{m}, \mathrm{Si}(\mathrm{CHMe})_{3}\right), 2.35$ $\left(1 \mathrm{H}, \mathrm{d}, J 17.5, \mathrm{C}(3) H_{\mathrm{A}}\right), 2.81\left(1 \mathrm{H}, \mathrm{d}, J 17.5, \mathrm{C}(3) H_{\mathrm{B}}\right), 3.75-3.80(1 \mathrm{H}, \mathrm{m}, \mathrm{C}(6) H), 3.89-3.98(3 \mathrm{H}, \mathrm{m}, \mathrm{C}(5) H$, $\left.\mathrm{C}\left(1^{\prime}\right) H_{2}\right), 4.09-4.14(1 \mathrm{H}, \mathrm{m}, \mathrm{C}(4) H), 4.17(1 \mathrm{H}$, br s, $\mathrm{OH}), 4.23\left(1 \mathrm{H}\right.$, br s, OH), $6.28\left(1 \mathrm{H}\right.$, br s, NH); $\delta_{\mathrm{C}}(125$ $\left.\mathrm{MHz}, \mathrm{CDCl}_{3}, 139 \mathrm{mM}\right)^{31} 11.7\left(\mathrm{Si}\left(\mathrm{CHMe}_{2}\right)_{3}\right), 17.9\left(\mathrm{Si}(\mathrm{CHMe})_{3}\right), 35.8(C(3)), 53.3(C(6)), 64.2\left(C\left(1^{\prime}\right)\right), 66.9$ $(C(4)), 68.1(C(5)), 171.6(C(2)) ; \mathrm{m} / z\left(\mathrm{ESI}^{+}\right) 318\left([\mathrm{M}+\mathrm{H}]^{+}, 72 \%\right), 340\left([\mathrm{M}+\mathrm{Na}]^{+}, 100 \%\right) ; \mathrm{HRMS}\left(\mathrm{ESI}^{+}\right)$ $\mathrm{C}_{15} \mathrm{H}_{32} \mathrm{NO}_{4} \mathrm{Si}^{+}\left([\mathrm{M}+\mathrm{H}]^{+}\right)$requires 318.2095; found 318.2090.

\subsubsection{X-ray crystal structure determination for 25}

Data were collected using an Oxford Diffraction SuperNova diffractometer with graphite monochromated $\mathrm{Cu}-\mathrm{K} \alpha$ radiation using standard procedures at $250 \mathrm{~K}$. The structures were solved by direct methods (SIR92); all non-hydrogen atoms were refined with anisotropic thermal parameters. Hydrogen atoms were added at idealised positions. The structures were refined using CRYSTALS. ${ }^{18,32}$

X-ray crystal structure data for $25\left[\mathrm{C}_{15} \mathrm{H}_{31} \mathrm{NO}_{4} \mathrm{Si}\right]: M=317.50$, orthorhombic, space group $P 2{ }_{1} 2{ }_{1} 2$,

$a=32.2727(12) \AA, b=11.3243(4) \AA, c=10.2112(4) \AA, V=3731.8(2) \AA^{3}, Z=8, \mu=1.228 \mathrm{~mm}^{-1}$, colourless block, crystal dimensions $=0.14 \times 0.16 \times 0.21 \mathrm{~mm}^{3}$. A total of 6959 unique reflections were 
measured for $4<\theta<76$ and 6933 reflections were used in the refinement. The final parameters were $w R_{2}=0.277$ and $R_{1}=0.105[I>-3.0 \sigma(I)]$, with Flack enantiopole $=+0.10(8) .{ }^{19}$

\subsection{1,2,5-Trideoxy-1,5-imino-D-lyxo-hexitol [D-4-epi-fagomine] 3}

$\mathrm{BH}_{3} \cdot \mathrm{SMe}_{2}(2.0 \mathrm{M}$ in THF, $1.14 \mathrm{~mL}, 2.27 \mathrm{mmol})$ was added to a solution of $24(103 \mathrm{mg}, 0.324 \mathrm{mmol},>95: 5$ dr) in THF (4 mL) at $0{ }^{\circ} \mathrm{C}$ and stirred at $0{ }^{\circ} \mathrm{C}$ for 30 min then warmed to rt over $2 \mathrm{~h}$ then $3 \mathrm{M}$ aq $\mathrm{NaOH}(2$ $\mathrm{mL})$ and $30 \%$ aq $\mathrm{H}_{2} \mathrm{O}_{2}(2 \mathrm{~mL})$ were added and the resultant mixture was heated at $85{ }^{\circ} \mathrm{C}$ for $1 \mathrm{~h}$ then cooled to $0{ }^{\circ} \mathrm{C} .6 \mathrm{M}$ aq $\mathrm{HCl}(5 \mathrm{~mL})$ was added and the resultant mixture was allowed to warm to $\mathrm{rt}$ over $18 \mathrm{~h}$ then the volatiles were removed in vacuo and the aqueous layer subjected to ion exchange chromatography (DOWEX 50WX8-200, eluent 1.0 M aq NH $\mathrm{NH}_{4} \mathrm{OH}$ ). Purification via flash column chromatography (eluent $\mathrm{CH}_{2} \mathrm{Cl}_{2} / \mathrm{MeOH} / 35 \%$ aq $\mathrm{NH}_{4} \mathrm{OH}, 50: 50: 1$ ) gave D-4-epi-fagomine 3 as a colourless viscous oil (22 mg, 46\%,

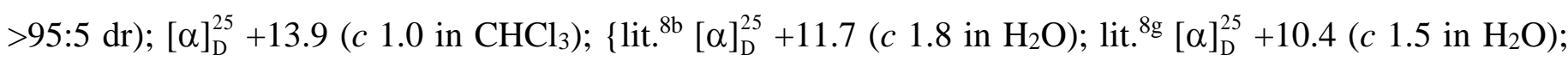
lit. $^{8 \mathrm{~d}}[\alpha]_{\mathrm{D}}^{22}+10.2\left(c 1.42\right.$ in $\left.\mathrm{H}_{2} \mathrm{O}\right)$; lit. $^{3}[\alpha]_{\mathrm{D}}+19\left(c 0.46\right.$ in $\left.\left.\mathrm{H}_{2} \mathrm{O}\right)\right\} ; \delta_{\mathrm{H}}\left(400 \mathrm{MHz}, \mathrm{D}_{2} \mathrm{O}\right)^{33} 1.92-2.10(2 \mathrm{H}, \mathrm{m}$, $\left.\mathrm{C}(2) H_{2}\right), 3.08\left(1 \mathrm{H}, \mathrm{td}, J 13.2,3.9, \mathrm{C}(1) H_{\mathrm{A}}\right), 3.34(1 \mathrm{H}, \mathrm{ddd}, J 8.5,5.1,1.2, \mathrm{C}(5) H), 3.47(1 \mathrm{H}, \mathrm{ddd}, J 13.2,4.6$, 2.4, C(1) $\left.H_{\mathrm{B}}\right), 3.79-3.94\left(3 \mathrm{H}, \mathrm{m}, \mathrm{C}(3) H, \mathrm{C}(6) H_{2}\right), 4.08-4.10(1 \mathrm{H}, \mathrm{m}, \mathrm{C}(4) H) ; \delta_{\mathrm{C}}\left(100 \mathrm{MHz}, \mathrm{D}_{2} \mathrm{O}\right)^{33} 27.0$ $(C(2)), 45.2(C(1)), 62.4(C(6)), 63.0(C(5)), 68.9(C(4)), 70.4(C(3)) ; m / z\left(\mathrm{ESI}^{+}\right) 148\left([\mathrm{M}+\mathrm{H}]^{+}, 100 \%\right)$; HRMS $\left(\mathrm{ESI}^{+}\right) \mathrm{C}_{6} \mathrm{H}_{14} \mathrm{NO}_{3}{ }^{+}\left([\mathrm{M}+\mathrm{H}]^{+}\right)$requires 148.0968; found 148.0969. A sample of 3 was dissolved in $\mathrm{CHCl}_{3} /{ }^{\mathrm{P} O \mathrm{PH}}(3: 1)$ and $2.0 \mathrm{M}$ aq $\mathrm{NaOH}$, and the aqueous layer was extracted with $\mathrm{CHCl}_{3} / \mathrm{PrOH}(3: 1)$, then the combined organic extracts were concentrated in vacuo to give $\mathbf{3}$ as a colourless viscous oil; $\delta_{\mathrm{H}}(400$ $\left.\mathrm{MHz}, \mathrm{D}_{2} \mathrm{O}\right)$ 1.64-1.72 (2H, m, C(2)H $\left.H_{2}\right), 2.56\left(1 \mathrm{H}, \mathrm{td}, J 12.5,4.3, \mathrm{C}(1) H_{\mathrm{A}}\right), 2.65-2.71(1 \mathrm{H}, \mathrm{m}, \mathrm{C}(5) H)$, 3.00-3.08 (1H, m, C(1)H $\left.H_{\mathrm{B}}\right), 3.60-3.66\left(2 \mathrm{H}, \mathrm{m}, \mathrm{C}(6) H_{2}\right), 3.70-3.76(1 \mathrm{H}, \mathrm{m}, \mathrm{C}(3) H), 3.88-3.92(1 \mathrm{H}, \mathrm{m}$, $\mathrm{C}(4) H) ; \delta_{\mathrm{C}}\left(100 \mathrm{MHz}, \mathrm{D}_{2} \mathrm{O}\right) 30.5(C(2)), 45.7(C(1)), 61.7(C(5)), 64.7(C(6)), 70.6(C(4)), 72.9(C(3))$.

\subsection{1,2,5-Trideoxy-1,5-imino-D-xylo-hexitol [D-5-epi-fagomine] 4}

$\mathrm{BH}_{3} \cdot \mathrm{SMe}_{2}(2.0 \mathrm{M}$ in THF, $1.38 \mathrm{~mL}, 2.76 \mathrm{mmol})$ was added to a solution of 25 (125 mg, 0.394 mmol, >95:5 dr) in THF $(4 \mathrm{~mL})$ at $0{ }^{\circ} \mathrm{C}$ and stirred at $0{ }^{\circ} \mathrm{C}$ for 30 min then warmed to rt over $2 \mathrm{~h}$ then $3 \mathrm{M}$ aq $\mathrm{NaOH}(2$ $\mathrm{mL})$ and $30 \%$ aq $\mathrm{H}_{2} \mathrm{O}_{2}(2 \mathrm{~mL})$ were added and the resultant mixture was heated at $85{ }^{\circ} \mathrm{C}$ for $1 \mathrm{~h}$ then cooled to $0{ }^{\circ} \mathrm{C} .6 \mathrm{M}$ aq $\mathrm{HCl}(5 \mathrm{~mL})$ was added and the resultant mixture was allowed to warm to $\mathrm{rt}$ over $18 \mathrm{~h}$ then the volatiles were removed in vacuo and the aqueous layer subjected to ion exchange chromatography (DOWEX 50WX8-200, eluent 1.0 M aq NH $4 \mathrm{OH}$ ). Purification via flash column chromatography (eluent $\mathrm{CH}_{2} \mathrm{Cl}_{2} / \mathrm{MeOH} / 35 \%$ aq $\left.\mathrm{NH}_{4} \mathrm{OH}, 25: 25: 1\right)$ gave D-5-epi-fagomine 4 as colourless viscous oil (25 mg, $43 \%$, 
$>95: 5 \mathrm{dr}) ;[\alpha]_{\mathrm{D}}^{25}+8.0\left(c 1.0\right.$ in $\left.\mathrm{H}_{2} \mathrm{O}\right) ;\left\{\right.$ lit. $^{8 \mathrm{~d}}[\alpha]_{\mathrm{D}}^{25}+13.4\left(c 0.32\right.$ in $\left.\mathrm{H}_{2} \mathrm{O}\right) ;$ lit. $^{27}[\alpha]_{\mathrm{D}}^{25}+12.1\left(c 0.33\right.$ in $\left.\left.\mathrm{H}_{2} \mathrm{O}\right)\right\}$; $\delta_{\mathrm{H}}\left(400 \mathrm{MHz}, \mathrm{D}_{2} \mathrm{O}\right)^{33} 1.81-1.89\left(1 \mathrm{H}, \mathrm{m}, \mathrm{C}(2) H_{\mathrm{A}}\right), 2.15-2.28\left(1 \mathrm{H}, \mathrm{m}, \mathrm{C}(2) H_{\mathrm{B}}\right), 3.21-3.32\left(2 \mathrm{H}, \mathrm{m}, \mathrm{C}(1) H_{2}\right)$, 3.51-3.59 (1H, m, C(5)H), $3.81\left(1 \mathrm{H}, \mathrm{dd}, J 12.2,8.7, \mathrm{C}(6) H_{\mathrm{A}}\right), 3.87\left(1 \mathrm{H}, \mathrm{dd}, J 12.2,5.0, \mathrm{C}(6) H_{\mathrm{B}}\right), 3.93-3.97$ $(1 \mathrm{H}, \mathrm{m}, \mathrm{C}(4) H), 4.01-4.07(1 \mathrm{H}, \mathrm{m}, \mathrm{C}(3) H) ; \delta_{\mathrm{C}}\left(100 \mathrm{MHz}, \mathrm{D}_{2} \mathrm{O}\right)^{33} 26.5(\mathrm{C}(2)), 41.7(C(1)), 58.7(C(5)), 62.4$ $(C(6)), 67.7(C(3)), 68.7(C(4)) ; m / z\left(\mathrm{ESI}^{+}\right) 148\left([\mathrm{M}+\mathrm{H}]^{+}, 100 \%\right) ; \mathrm{HRMS}\left(\mathrm{ESI}^{+}\right) \mathrm{C}_{6} \mathrm{H}_{14} \mathrm{NO}_{3}{ }^{+}\left([\mathrm{M}+\mathrm{H}]^{+}\right)$ requires 148.0968; found 148.0967. A sample of 4 was dissolved in $\mathrm{CHCl}_{3} / \mathrm{PrOH}(3: 1)$ and $2.0 \mathrm{M} \mathrm{aq} \mathrm{NaOH}$, and the aqueous layer was extracted with $\mathrm{CHCl}_{3} /{ }^{\mathrm{i}} \mathrm{PrOH}$ (3:1), then the combined organic extracts were concentrated in vacuo to give 4 as a colourless viscous oil; $\delta_{\mathrm{H}}\left(400 \mathrm{MHz}, \mathrm{D}_{2} \mathrm{O}\right) 1.58(1 \mathrm{H}, \mathrm{dq}, J$ 14.1, 4.2, $\left.\mathrm{C}(2) H_{\mathrm{A}}\right), 1.91-2.01\left(1 \mathrm{H}, \mathrm{m}, \mathrm{C}(2) H_{\mathrm{B}}\right), 2.81-2.88\left(2 \mathrm{H}, \mathrm{m}, \mathrm{C}(1) H_{2}\right) .3 .10(1 \mathrm{H}, \mathrm{td}, J 6.7,2.6, \mathrm{C}(5) H), 3.62-3.69$ $\left(2 \mathrm{H}, \mathrm{m}, \mathrm{C}(6) \mathrm{H}_{2}\right), 3.69-3.73(1 \mathrm{H}, \mathrm{m}, \mathrm{C}(4) H), 3.90\left(1 \mathrm{H}\right.$, app q, $J$ 4.3, C(3)H); $\delta_{\mathrm{C}}\left(100 \mathrm{MHz}, \mathrm{D}_{2} \mathrm{O}\right) 30.6(C(2))$, $41.4(C(1)), 58.1(C(5)), 63.5\left(C\left(1^{\prime}\right)\right), 70.5(C(4)), 71.5(C(3))$.

\section{References and Notes}

${ }^{1}$ Koyama, M.; Sakamura, S. Agric. Biol. Chem. 1974, 38, 1111.

${ }^{2}$ Asano, N.; Tomioka, E.; Kizu, H.; Matsui, K. Carbohydr. Res. 1994, 253, 235.

${ }^{3}$ Asano, N.; Oseki, K.; Kizu, H.; Matsui, K. J. Med. Chem. 1994, 37, 3701.

${ }^{4}$ Kato, A.; Asano, N.; Kizu, H.; Matsui, K.; Watson, A. A.; Nash, R. J. J. Nat. Prod. 1997, 60, 312.

${ }^{5}$ Molyneux, R. J.; Benson, M.; Wong, R. Y.; Tropea, J. E.; Elbein, A. D. J. Nat. Prod. 1988, 51, 1198.

${ }^{6}$ Asano, N.; Oseki, K.; Tomioka, E.; Kizu, H.; Matsui, K. Carbohydr. Res. 1994, 259, 243.

${ }^{7}$ Kato, A.; Hirokami, Y.; Kinami, K.; Tsuji, Y.; Miyawaki, S.; Adachi, I.; Hollinshead, J.; Nash, R. J.; Kiappes, J. L.; Zitzmann, N.; Cha, J. K.; Molyneux, R. J.; Fleet, G. W. J. Phytochemistry 2015, 111, 124.

${ }^{8}$ For example, see: (a) Espelt, L.; Parella, T.; Bujons, J.; Solans, C.; Joglar, J.; Delgado, A.; Clapés, P. Chem.-Eur. J. 2003, 9, 4887; (b) Kumar, K. S. A.; Rathee, J. S.; Subramanian, M.; Chattopadhyay, S. J. Org. Chem. 2013, 78, 7406; (c) Corkran, H. M.; Munneke, S.; Dangerfield, E. M.; Stocker, B. L.; Timmer, M. S. M. J. Org. Chem. 2013, 78, 9791; (d) Takahata, H.; Banba, Y.; Ouchi, H.; Nemoto, H.; Kato, A.; Adachi, I. J. Org. Chem. 2003, 68, 3603; (e) Kato, A.; Miyauchi, S.; Kato, N.; Nash, R. J.; Yoshimura, Y.; Nakagome, I.; Hirono, S.; Takahata, H.; Adachi, I. Bioorg. Med. Chem. 2011, 19, 3558; (f) van den Berg, R. J. B. H. N.; Wennekes, T.; Ghisaidoobe, A.; Donker-Koopman, W. E.; Strijland, A.; Boot, R. G.; van der Marel, G. A.; Aerts, J. M. F. G.; Overkleeft, H. S. ACS Med. Chem. Lett. 2011, 2, 519; (g) Kumari, N.; Reddy, B. G.; Vankar, Y. D. Eur. J. Org. Chem. 2009, 160. 
${ }^{9}$ Nojima, H.; Kimura, I.; Chen, F.-J.; Sugihara, Y.; Haruno, M.; Kato, A.; Asano, N. J. Nat. Prod. 1998, 61, 397.

${ }^{10}$ Asano, N.; Ishii, S.; Kizu, H.; Ikeda, K.; Yasuda, K.; Kato, A.; Martin, O. R.; Fan, J.-Q. Eur. J. Biochem. 2000, 267, 4179 .

11 (a) Davies, S. G.; Smith, A. D.; Price, P. D. Tetrahedron: Asymmetry 2005, 16, 2833; (b) Davies, S. G.; Fletcher, A. M.; Roberts, P. M.; Thomson, J. E. Tetrahedron: Asymmetry 2012, 23, 1111; (c) Davies, S. G.; Fletcher, A. M.; Roberts, P. M.; Thomson, J. E. Tetrahedron: Asymmetry 2017, 28, 1842.

12 Davies, S. G.; Figuccia, A. L. A.; Fletcher, A. M.; Roberts, P. M.; Thomson, J. E. Org. Lett. 2013, 15, 2042.

${ }^{13}$ For the preparation of the antipode, see: Abraham, E.; Brock, E. A.; Candela-Lena, J. I.; Davies, S. G.; Georgiou, M.; Nicholson, R. L.; Perkins, J. H.; Roberts, P. M.; Russell, A. J.; Sánchez-Fernández, E. M.; Scott, P. M.; Smith, A. D.; Thomson, J. E. Org. Biomol. Chem. 2008, 6, 1665.

14 (a) Davies, S. G.; Walters, I. A. S. J. Chem. Soc., Perkin Trans. 1 1994, 1129. (b) Davies, S. G.; Foster, E. M.; McIntosh, C. R.; Roberts, P. M.; Rosser, T. E.; Smith, A. D.; Thomson, J. E. Tetrahedron: Asymmetry 2011, 22, 1035.

${ }^{15}$ An authentic sample of aldehyde 10 was prepared in 63\% yield and >95:5 dr upon Swern oxidation of alcohol 9.

${ }^{16}$ A sample of the C(4)-epimer was also isolated in 20\% yield and >95:5 dr.

17 For a related example concerning direct lactonization upon hydrogenolysis of a $\delta$-amino ester, see: Garrido, N. M.; García, M.; Díez, D.; Sánchez, M. R.; Sanz, F.; Urones, J. G. Org. Lett. 2008, 10, 1687.

${ }^{18}$ Crystallographic data (excluding structure factors) for $\mathbf{1 6}$ and $\mathbf{2 5}$ have been deposited with the Cambridge Crystallographic Data Centre as supplementary publication numbers CCDC 1863200 and 1863201, respectively.

19 (a) Flack, H. D. Acta Crystallogr. Sect. A 1983, 39, 876. (b) Flack, H. D.; Bernardinelli, G. J. Appl. Crystallogr. 2000, 33, 1143.

${ }^{20}$ It was necessary to collect crystallographic data for both $\mathbf{1 6}$ and $\mathbf{2 5}$ at $250 \mathrm{~K}$ as the structures were modulated at lower temperatures.

${ }^{21}$ Fleet, G. W. J.; Smith, P. W. Tetrahedron Lett. 1985, 26, 1469.

${ }^{22}$ Fleet, G. W. J.; Fellows, L. E.; Smith, P. W. Tetrahedron 1987, 43, 979.

${ }^{23}$ Fleet, G. W. J.; Witty, D. R. Tetrahedron:Asymmetry 1990, 1, 119. 
${ }^{24}$ For example, see: (a) Davies, S. G.; Fletcher, A. M; Foster, E. M; Lee, J. A; Roberts, P. M.; Thomson, J. E. J. Org. Chem. 2013, 78, 2500. (b) Davies, S. G.; Fletcher, A. M.; Frost, A. B.; Lee, J. A.; Roberts, P. M.; Thomson, J. E. Tetrahedron 2013, 69, 8885. (c) Brambilla, M.; Davies, S. G.; Fletcher, A. M.; Hao, L.; Lv, L.; Roberts, P. M.; Thomson, J. E. Tetrahedron 2014, 70, 3491. (d) Archer, S. G.; Csatayová, K.; Davies, S. G.; Fletcher, A. M.; Roberts, P. M.; Thomson, J. E. Tetrahedron Lett. 2016, 57, 1270. (e) Buchman, M.; Csatayová, K.; Davies, S. G.; Fletcher, A. M.; Houlsby, I. T. T.; Roberts, P. M.; Rowe, S.; Thomson, J. E. J. Org. Chem. 2016, 81, 4907. For an alternative strategy for C(2)-epimerisation, see: Bannage, M. E.; Burke, A. J.; Davies, S. G.; Goodwin, C. J. Tetrahedron: Asymmetry 1994, 5, 203.

${ }^{25}$ A diastereoisomerically enriched sample of 22 and 23 (30:70 dr) was treated under the same conditions and gave a $\sim 30: 70$ mixture of lactams $\mathbf{2 4}$ and 25, respectively, which were isolated in 7 and $43 \%$ yield, and $>95: 5 \mathrm{dr}$ in each case.

${ }^{26}$ The ${ }^{1} \mathrm{H}$ and ${ }^{13} \mathrm{C}$ NMR data for D-4-epi-fagomine 3 and D-5-epi-fagomine 4 were, however, shown to vary in the presence of trace impurities of acid, which thus reconciled discrepancies between different literature reports.

${ }^{27}$ Kundu, P. K.; Ghosh, S. K. Tetrahedron: Asymmetry 2011, 22, 1090.

${ }^{28}$ Pangborn, A. B.; Giardello, M. A.; Grubbs, R. H.; Rosen, R. K.; Timmers, F. J. Organometallics 1996, 15, 1518.

${ }^{29}$ Abraham, E.; Davies, S. G.; Millican, N. L.; Nicholson, R. L.; Roberts, P. M.; Smith, A. D. Org. Biomol. Chem. 2008, 6, 1655.

${ }^{30}$ Esteve, J.; Jimenez, C.; Nebot, J.; Velasco, J.; Romea, P.; Urpí, F. Tetrahedron 2011, 67, 6045.

${ }^{31}$ The ${ }^{1} \mathrm{H}$ and ${ }^{13} \mathrm{C}$ NMR chemical shifts were seen to be concentration dependant so were recorded at two different concentrations.

${ }^{32}$ Betteridge, P. W.; Carruthers, J. R.; Cooper, R. I.; Prout, C. K.; Watkin, D. J. J. Appl. Crystallogr. 2003, $36,1487$.

${ }^{33}$ Chemical shifts were measured downfield from sodium 3-(trimethylsilyl)propanoate. 